\title{
Non-canonical glutamine transamination metabolism sustains efferocytosis by coupling oxidative stress buffering to oxidative phosphorylation
}

Johanna Merlin

Inserm

Stoyan Ivanov

Washington University in St.Louis

Adélie Dumont

Centre Méditerranéen de Médecine Moléculaire (C3M)

Alexey Sergushichev

ITMO University https://orcid.org/0000-0003-1159-7220

Julie Gall

inserm

Marion Stunault

inserm https://orcid.org/0000-0002-4298-5114

Marion Ayrault

Inserm

Nathalie Vaillant

Inserm

Amanda Swain

Washington University in St. Louis https://orcid.org/0000-0003-0683-368X

Francois Orange

Centre Commun de Microscopie Appliquée (CCMA)

Alexandre Gallerand

Inserm

Thierry Berton

Inserm/INRA

Jean-Charles Martin

Inserm/INRA https://orcid.org/0000-0002-2870-0012

\section{Stefania Carobbio}

Faculty of Medicine, University of Geneva

Justine Masson

Columbia University 
Inna Gaisler-Salomon

Columbia University

Pierre Maechler

University of Geneva https://orcid.org/0000-0002-2005-1433

\section{Stephen Rayport}

Columbia University

Judith Sluimer

Maastricht University Medical Centre https://orcid.org/0000-0002-0269-4582

\section{Erik Biessen}

Maastricht University Medical Center

\section{Rodolphe Guinamard}

Inserm/cnrs

\section{Emmanuel Gautier}

Inserm

\section{Edward Thorp}

Northwestern University

\section{Maxim Artyomov}

Washington University in St.Louis https://orcid.org/0000-0002-1133-4212

Laurent Yvan-Charvet ( $\square$ yvancharvet@unice.fr )

Inserm https://orcid.org/0000-0002-7748-4942

\section{Article}

Keywords: Macrophage, Glutaminolysis, Efferocytosis, Interleukin-4 and Atherosclerosis

Posted Date: October 8th, 2020

DOI: https://doi.org/10.21203/rs.3.rs-85098/v1

License: (9) (i) This work is licensed under a Creative Commons Attribution 4.0 International License. Read Full License

Version of Record: A version of this preprint was published at Nature Metabolism on October 14th, 2021. See the published version at https://doi.org/10.1038/s42255-021-00471-y. 
Non-canonical glutamine transamination metabolism sustains efferocytosis by coupling oxidative stress buffering to oxidative phosphorylation.

Johanna Merlin ${ }^{1^{*}}$, Stoyan Ivanov ${ }^{1^{*}}$, Adélie Dumont ${ }^{1}$, Alexey Sergushichev ${ }^{2}$, Julie Gall ${ }^{1}$, Marion Stunault ${ }^{1}$, Marion Ayrault ${ }^{1}$, Nathalie Vaillant ${ }^{1}$, Amanda Swain $^{3}$, Francois Orange ${ }^{4}$, Alexandre Gallerand ${ }^{1}$, Thierry Berton ${ }^{5}$, Jean-Charles Martin ${ }^{5}$, Stefania Carobbio ${ }^{6}$, Justine Masson ${ }^{7}$, Inna Gaisler-Salomon ${ }^{7}$, Pierre Maechler ${ }^{6}$, Stephen Rayport ${ }^{7}$, Judith C. Sluimer $^{8}$, Erik A. L. Biessen ${ }^{8}$, Rodolphe R. Guinamard ${ }^{1}$, Emmanuel L. Gautier ${ }^{9}$, Edward B. Thorp ${ }^{10}$, Maxim N. Artyomov ${ }^{3}$ and Laurent Yvan-Charvet ${ }^{1}$

*These authors contributed equally to this work

${ }^{1}$ Institut National de la Santé et de la Recherche Médicale (Inserm) U1065, Université Côte d'Azur, Centre Méditerranéen de Médecine Moléculaire (C3M), Atip-Avenir, Fédération Hospitalo-Universitaire (FHU) Oncoage, 06204 Nice, France (J.M., S.I., J.G., M.S., M.A., A.G., R.G., L.Y.C.)

${ }^{2}$ Computer Technologies Department, ITMO University, Saint Petersburg, Russia (A.S.)

${ }^{3}$ Department of Pathology and Immunology, Washington University School of Medicine, St. Louis, MO, USA (A.S., M.N.A.)

${ }^{4}$ Université Côte d'Azur, Centre Commun de Microscopie Appliquée (CCMA), 06108 Nice, France (F.O)

${ }^{5}$ Centre de Recherche Cardiovasculaire et Nutritionnelle (C2VN), INSERM, Institut National de la Recherche Agricole (INRA), BioMet, Aix-Marseille University, Marseille, France (T.B., J.C.M.)

${ }^{6}$ Department of Cell Physiology and Metabolism; Faculty Diabetes Centre, University of Geneva Medical Centre, Geneva, Switzerland (S.C., P.M.)

${ }^{7}$ Department of Psychiatry, Columbia University, USA; Department of Molecular Therapeutics, NYS Psychiatric Institute, USA (J.M., I.G.S., S.R)

${ }^{8}$ Department of Pathology, Cardiovascular Research Institute Maastricht, Maastricht University Medical Center, Maastricht, the Netherlands (J.C.S., E.A.L.B.)

${ }^{9}$ Sorbonne Université, INSERM, UMR_S 1166 ICAN, F-75013 Paris, France (E.L.G).

${ }^{10}$ Department of Pathology, Northwestern University, Feinberg School of Medicine, Chicago, Illinois 60611, USA (E.B.T.)

Address correspondence to LYC: yvancharvet@unice.fr

Key words: Macrophage, Glutaminolysis, Efferocytosis, Interleukin-4 and Atherosclerosis 


\section{Summary}

Macrophages rely on tightly integrated metabolic rewiring to clear dying neighboring cells by efferocytosis during homeostasis and disease. Here, we reveal glutaminase (Gls) 1-mediated glutaminolysis is critical to promote apoptotic cell clearance by macrophages during homeostasis. In addition, impaired macrophage glutaminolysis exacerbated atherosclerosis, a condition during which efficient apoptotic cell debris clearance is critical to limit disease progression. Gls1 expression strongly correlated with atherosclerotic plaque necrosis in patients with cardiovascular diseases. High-throughput transcriptional and metabolic profiling revealed that macrophage efferocytic capacity rely on a non-canonical transaminase pathway, independent from the traditional requirement of glutamate dehydrogenase (Glud1) to fuel aketogulatrate-dependent immunometabolism. This pathway was necessary to meet the unique requirements of efferocytosis for cellular detoxification and high energy cytoskeletal rearrangements. Thus, we uncovered a novel role for non-canonical glutamine metabolism for efficient clearance of dying cells and maintenance of tissue homeostasis during health and disease.

\section{Highlights}

- Macrophage glutaminase (Gls) 1-mediated glutaminolysis facilitates homeostatic apoptotic cell clearance.

- Macrophage glutaminolysis inhibition led to defective efferocytosis in atherosclerotic lesions.

- Glud1 deficiency does not recapitulate the defective efferocytosis of Gls1 deficient macrophages.

- High-throughput profiling revealed that efferocytosis relied on a non-canonical transamination metabolism of glutamine to support the high energy actin dynamics. 


\section{Introduction}

Clearance of apoptotic cells (ACs) by macrophages (i.e, efferocytosis) prevent the leakage of dying cellular contents to maintain tissue integrity in normal physiology (Kojima et al., 2017; Elliott and Ravichandran, 2016). Impaired efferocytosis in disease can have multiple causes, but defects in the internalization of multiple ACs, a process termed 'continual efferocytosis', has emerged as a culprit of many chronic inflammatory diseases such as atherosclerosis (Wang et al., 2017; Yurdagul et al., 2020). Resolution and repair processes also require the cytokine interleukin-4 (IL-4) (Bosurgi et al., 2017) and an efficient cellular metabolic reprogramming to sustain continual efferocytosis (Han and Ravichandran, 2011). However, the understanding of metabolism during efferocytosis on a molecular level is still poorly understood and fundamentally and clinically relevant.

Glutamine metabolism is considered as a 'fuel for the immune system' and is routinely used as a component for clinical supplementation in trauma patients. Glutamine is initially hydrolyzed into glutamate by the glutaminase Gls1 in a metabolic process called glutaminolysis (DeBerardinis and Cheng, 2010; O'Neill and Pearce, 2016), which is the most upregulated pathway in reparative-type macrophages induced by IL-4 dependent alternative activation (Jha et al., 2015). Yet, whether glutaminolysis supports the functional metabolic reprogramming of efferocytosis beyond glucose and fatty acid metabolism remains unknown (Morioka et al., 2018; Zhang et al., 2019).

Here, we tested the hypothesis that glutaminolysis plays a critical role in macrophage efferocytic capacity. Mice lacking macrophage Gls1 exhibited defective efferocytosis in vivo and in vitro under reparative conditions or upon successive rounds of exposure to dying cells. The clinical and pathologic relevance of glutaminolysis in this process was exemplified with the inverse association with the accumulation of necrotic core in human and murine atherosclerotic lesions. Mechanistically, glutaminolysis channeled glutamate into the malateaspartate shuttle through an aspartate aminotransferase (GOT)-dependent transamination process to meet the demand for cellular detoxification and cytoskeletal rearrangements and achieve efficient efferocytosis. 


\section{Results}

Gls1-mediated glutaminolysis in reparative and resolving-type macrophages orchestrates optimal efferocytosis. To investigate metabolic roles of macrophage glutaminolysis during clearance functions, mice bearing a conditional allele for glutaminase 1 $\left(G / s 1^{f / f f}\right)$ in macrophages were generated by crossing to Lysozyme M-Cre transgenic mice (LysMCre). LysM-Cre $x$ Gls $1^{\text {fl/f }}$ peritoneal cavity macrophages (Mac ${ }^{\Delta \mathrm{Gls} 1} \mathrm{PCMs}$ ) efficiently deleted G/s1 and presented with a 2-fold decrease in cellular glutamate levels as compared to controls (Fig. 1a). Glutaminolysis can control the balance between cellular life and death in vitro through mTORC1 pathway or ferroptosis, respectively (Dixon et al., 2012; Nicklin et al., 2009). Although G/s1 was expressed to various levels in PCMs, bone marrow (BM) monocytes/macrophages, red pulp macrophages (RPMs), Kupffer cells and microglia (Fig. S1a), their numbers were unaltered in in Mac ${ }^{\Delta \mathrm{Gls} 1}$ mice (Fig. S1b). Consistently, proliferation and apoptosis were similar in Mac ${ }^{\Delta \mathrm{Gls} 1} \mathrm{PCM}$ compared to control cells (Fig. S1C). Levels of phospho-S6 and Myc protein, mTORC1 downstream targets, were also unchanged in these cells (Fig. S1d) along with absence of transcriptional regulation of rapamycin (mTOR), hypoxia or ferroptosis targets (Fig. S1e). These data strongly suggested that glutaminolysis is not required for macrophage maintenance or development at the steady state. Consistent with the strong enrichment in glutaminolytic processes in IL-4 treated reparative macrophages (Jha et al., 2015), we observed an impaired induction of canonical alternatively activated genes (Clec10a, Tgm2, Arg1, Stab1, Sepp1) (Fig. S1f) and cell surface expression of CD206 and CD301 (Fig. S1g) in Mac ${ }^{\Delta G \mid s 1}$ PCMs. Additionally, challenging PCMs with either IL-4, apoptotic cells (ACs) or IL4 and ACs together revealed that Gls1-dependent glutaminolysis was required for efficient efferocytosis in reparative macrophages. Indeed, we measured a $28 \%$ phagocytic index reduction of Mac ${ }^{\Delta \text { Gls1 }}$ reparative PCMs after 45 min exposure to apoptotic thymocytes (Fig. 1b). Similar findings were observed using bone marrow-derived macrophages (BMDMs) (Fig. S1h). Importantly, overexpression of Gls1 in BMDMs using lentiviral particles enhanced efferocytosis in un-stimulated control cells and rescued the defective efferocytosis observed in reparative $\mathrm{Mac}^{\Delta \mathrm{Gls} 1}$ macrophages (Fig. S1h). To test the impact of glutaminolysis on efferocytosis during heightened metabolic challenge, we subjected Gls1-deficient macrophages to successive rounds of AC clearance, which mimics tissue states of high cell turnover (Han and Ravichandran, 2011). We performed a validated two-stage efferocytosis experiment in which BMDMs were first incubated for $45 \mathrm{~min}$ with unlabeled ACs and, after AC removal and a onehour interval, incubated with a second round of labelled ACs (Wang et al., 2017). Under these conditions, the percentage of resolving macrophages that had internalized the second round of ACs was significantly decreased in Gls1-deficient macrophages relative to controls (Fig. 1c). To test the in vivo significance, we intravenously injected fluorescently labeled ACs into controls and Mac ${ }^{\Delta \mathrm{Gls} 1}$ mice. Gls1 deficiency reduced the ability of $\mathrm{CD} 11 \mathrm{~b}^{\text {lo }} \mathrm{F} 4 / 80^{\text {high }}$ Kupffer cells (KCs) in the liver and to some extent $\mathrm{CD} 206^{\text {high }} \mathrm{F} 4 / 80^{\text {high }}$ red pulp macrophages (RPMs) in the spleen to internalize labeled ACs one hour after injection (Fig. 1d). These findings provide the first in vivo genetic evidence of the pivotal role of Gls1-dependent glutaminolysis during macrophage phagocytic clearance.

Glutaminolysis regulates lesional macrophage efferocytosis in atherosclerosis. To determine the relevance of these observations during disease, we next tested Gls1 requirements in a mouse model of atherosclerosis, in which defective lesional dead cell clearance translates into unstable necrotic core within plaques (Kojima et al., 2017; Tabas, 2010). Taking advantage of publicly available gene expression datasets of western diet (WD) fed wild-type (WT) and ApoE-deficient mice (Grabner et al., 2009), and computational analysis of metabolic networks (Jha et al., 2015), we first observed a downregulation of Gls1 expression in atheromatous plaques. This was part of a dysregulated amino acid catabolism module making up a densely connected core with a more global metabolic transcriptome signature (Fig. S2a). Lower Gls1 expression correlated with an imbalance in markers of inflammatory and reparative macrophages (Heatmap, Fig. S2a). To monitor glutaminolysis activity in atherosclerotic plaque, we next assessed radiolabeled $\left[{ }^{14} \mathrm{C}\right]$ glutamine uptake and hydrolysis 
in aortas extracted from WT and $\mathrm{ApoE}^{-/-}$mice fed on WD for 6 weeks. Biomolecular imaging revealed higher glutamine incorporation into plaque areas enriched in macrophages (Fig. S2b). Nevertheless, quantification of $\left[{ }^{14} \mathrm{C}\right]$ glutamine conversion into glutamate, after separation by thin-layer chromatography, confirmed lower glutaminase-dependent metabolism in atherosclerotic aorta (Fig. S2c). Next, Mac ${ }^{\Delta \mathrm{Gls} 1}$ mice were crossed onto an atherosclerotic ApoE-deficient background. After WD feeding (12 weeks), there was an approximately 1.7-fold increase in atherosclerosis plaque area in the aortic sinus compared to co-housed control littermates (Fig. 2a). Oil red $\mathrm{O}$ staining in descending aortas confirmed higher plaque burden in Apoe $^{-/-}$Mac $^{\Delta G l s 1}$ mice (Fig. 2b) despite similar plasma lipid levels (Fig. S2d). Increased plaque area was also visualized in an independent cohort of $A p o e^{-/} \mathrm{Mac}^{\Delta \mathrm{Gls} 1}$ mice using noninvasive ultrasound imaging (Fig. 2c). These effects were specific to macrophage glutaminolysis since hematopoietic Gls1-deficiency (i.e, generation of Mx1-Cre $\times$ G/s $1^{f / f l}$ mice onto the atherosclerotic Apoe ${ }^{-/}$background) recapitulated similar accelerated atherosclerosis relative to control animals (Fig. S2e). Further characterization of atherosclerotic lesions in $\mathrm{ApoE}^{-/-} \mathrm{Mac}^{\Delta \mathrm{Gls} 1}$ mice revealed complex plaques containing macrophages (Fig. S2f). We did not observe significant change in Ki67-positive proliferative cells in Apoe ${ }^{-/}$Mac $^{\Delta G l s 1}$ mice (Fig. S2f). In contrast, necrotic cores were typified by a greater proportion of TUNEL-positive ACs and a reduced ratio of macrophage associated ACs versus to free ACs, a hallmark of defective efferocytosis (Fig. 2d). To further support that glutaminolyis enables optimal efferocytosis in this atherogenic model, we injected interperitoneally $A p o e^{-/-} \mathrm{Mac}^{\Delta \mathrm{Gls} 1}$ mice with fluorescent dyelabeled apoptotic lymphocytes and examined macrophage efferocytic capacity one hour later.

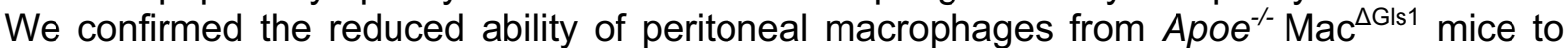
efficiently perform efferocytosis (Fig. S2g). To examine clinical significance, we investigated whether Gls1 expression correlated with human atherosclerotic plaque complexity. We found a reduced Gls1 expression in unstable vs. stable human carotid artery plaque (Fig. 2e). Moreover, we uncovered a positive correlation between Gls1 expression and the M2-specific marker $\mathrm{Arg}^{+}$(Fig. 2e). By contrast, Gls1 levels inversely correlated with plaque size, intraplaque hemorrhage (IPH) and necrotic core composition (Fig. 2e), key markers of atherosclerosis severity. Taken together, our findings demonstrate that Gls1 contributes to AC engulfment in the pathological process of human and murine atherosclerosis.

Glutaminolysis controls apoptotic corpses engulfment by macrophages. As reported above, IL-4 treated macrophage relied on Gls1 to achieve full efferocytic capacity. We first found that intracellular G/s1 mRNA expression was similarly upregulated by IL-4 stimulation or AC ingestion in a validated gene expression dataset (Bosurgi et al., 2017) (Fig. S3a). We confirmed this upregulation in a time course experiment (Fig. S3b), paralleling a drop in cellular glutamine to glutamate ratio (Fig. S3c). Thus, glutaminolysis is intrinsically regulated in resolving macrophages. To evaluate how IL-4 might couple macrophage glutaminolysis to efferocytosis, we next performed an RNA sequencing (RNA-seq) analysis of PCMs stimulated by IL-4 in vivo. When compared to control PCMs, G/s1-deficient cells had similar transcriptional regulation of genes involved in the sensing of soluble mediators attracting the phagocytes to ACs ('smell' or 'find-me' signals), the direct contact and recognition of ACs by ligand-receptor interactions ('taste' or 'eat me' signals) and the corpse internalization and processing ('ingestion' and 'digestion' processes) (Han and Ravichandran, 2011) (Fig. S3d). Thus, glutaminolysis does not control the transcriptional efferocytic reprograming in reparative macrophages. To next distinguish whether defective glutaminolysis impact AC binding or internalization, we incubated macrophages with cytochalasin D, an actin polymerization inhibitor (Yurdagul et al., 2020). In the cytochalasin D-treated cells, macrophage-AC association was similar among control and reparative Mac ${ }^{\Delta \mathrm{Gls} 1} \mathrm{BMDMs}$ (Fig. 3a), indicating that Gls1 promote AC internalization, not binding. These findings suggest a defect in the dynamic rearrangement of actin to engulf corpse during phagosome sealing (Han and Ravichandran, 2011). This energy-intensive process can be regulated by polymerization and depolymerization steps that can be accelerated by an ATP-dependent nucleation phase of new actin filaments and regulated by small GTPases such as Rac1 and Cdc42 (Fig. S3e)(Caron et al., 1998). Although Cdc42 mRNA expression was slightly down-regulated in reparative Gls1 deficient 
macrophages, neither Rac1 expression (Fig. S3f) nor the newly identified Rac1 transcriptional regulator GTP-exchange factor Dbl was affected (Fig. S3g)(Yurdagul et al., 2020). Similar gene expression of polymerization/depolymerization regulators was also observed (Fig. S3f). Thus, actin cytoskeletal rearrangement was unlikely mediated by transcriptional regulation. Nevertheless, we observed a slower polarization of G- to F-actin in reparative Mac ${ }^{\Delta G \mid s 1}$ macrophages (Fig. S3h) along with reduced activity of Cdc42 and Rac1 (Fig. S3i). Consistently, transmission electron microscopy revealed less membrane ruffling surrounding the Mac ${ }^{\Delta \mathrm{Gls} 1}$ BMDMs (Fig. 3b). Finally, phalloidine immunostaining, $45 \mathrm{~min}$ after efferocytosis, revealed a general decrease in the amount of F-actin staining at the leading edge of membrane ruffle formation surrounding the apoptotic cells (Fig. 3c). Thus, we identify that glutaminolysis is essential to meet the demand for high energy cytoskeletal rearrangement that facilitates the process of corpse engulfment.

\section{Glutaminolysis potentiates efferocytosis by fueling mitochondrial oxidative} phosphorylation (OXPHOS). To identify how glutamine-dependent pathways could meet the high energy demand of reparative macrophages, we traced ${ }^{13} \mathrm{C}$-labeled glutamine intracellular fate and quantified several metabolites and by-products by liquid chromatography-mass spectrometry. Labeling distribution analyses revealed lower ${ }^{13} \mathrm{C}$ incorporation into TCA metabolites (succinate, malate, aconitase) and ATP in reparative $\mathrm{Mac}^{\Delta \mathrm{Gls} 1} \mathrm{BMDMs}$, supporting a partially defective TCA cycling in these cells (Fig. 3d). By contrast, branched-chain amino acid (BC-AA) catabolism and urea cycle turnover were not perturbed in Mac ${ }^{\Delta G \mid s 1} B M D M s$ and thus unlikely participated to the lower energy-dependent engulfment process of G/s1-deficient macrophages (Fig. S3j to S3n). We next provided evidence of reduced basal oxygen consumption rate (OCR) and maximal respiration after FCCP (carbonyl cyanide 4[trifluoromethoxy] phenylhydrazone) treatment in Mac ${ }^{\Delta \mathrm{Gls} 1} \mathrm{PCM}$ and BMDMs in comparison to control cells (Fig. S3o and S3p). These effects occurred independently of fluctuation in extracellular acidification rate (ECAR), a surrogate of glycolytic activity (Fig. S3q). Flux measurements also showed that resting and reparative Mac ${ }^{\Delta \mathrm{Gls} 1}$ BMDMs fed ACs exhibited a decrease in maximal respiration response (Fig. $\mathbf{3 e}$ and $\mathbf{3 f}$ ). These effects were associated with a drop in ATP production reflecting an inability to fully respond to the increase in energy demand (Fig. S3r). Similar to IL-4 stimulation, basal OCR and maximal respiration response were also reduced in G/s1-deficient macrophages during continued clearance of ACs (Fig. $\mathbf{3 g}$ ). The observed altered mitochondrial metabolism was associated with higher total and mitochondrial ROS levels in resting and reparative Mac $^{\Delta \mathrm{Gls} 1}$ BMDMs (Fig. S3s) suggesting a reverse electron transport (RET) by the mitochondrial electron transport chain (ETC). To dissect the origin of the defective OCR in G/s1-deficient macrophages and its causal role on efferocytosis, we used ETC pharmacological inhibitors or supplemented Mac ${ }^{\Delta \mathrm{Gls} 1}$ BMDMs with mitochondrial ROS scavengers (Fig. 3h). Indeed, mitochondrial redox status has been previously shown to metabolically program macrophage effector function (Jais et al., 2014; Vats et al., 2006). Mitochondrial ROS scavenging by the superoxide dismutase mimetic (Tempol) or the mitochondria-targeted antioxidant coenzyme Q10 (MitoQ) was insufficient to rescue the defective OCR of reparative $M \mathrm{Mc}^{\Delta \mathrm{Gls} 1} \mathrm{BMDMs}$, suggesting that mitochondrial ROS production are most likely a consequence, rather than the cause, of the metabolic rewiring (Fig. 3i). These treatments did also not improve the efferocytic index in Mac ${ }^{\Delta G / s 1} B M D M s$ (Fig. 3j). By contrast, we observed that direct OCR inhibition by targeting mitochondrial complex II (i.e, succinate dehydrogenase (SDH)) with 3-Nitropropionic acid (3NPA) or complex III with antimycin A reduced efferocytosis in control cells to the levels of reparative Mac ${ }^{\Delta \mathrm{Gls} 1} \mathrm{BMDMs}$ (Fig. 3j). Similar findings were observed during continued clearance of ACs (Fig. S3t). Consistent with the reduced OCR and pro-inflammatory properties of Risp knockout macrophages (Zhang et al., 2019), we also confirmed the genetic requirement of complex III to efferocytosis and continued clearance of ACs (Fig. S3u). Together, these findings reveal that glutaminolysis couples mitochondrial oxidative phosphorylation to ATP production for efficient efferocytosis. 
Topological analyses predict how glutaminolysis rewires transcriptional metabolic networks in reparative-type macrophages. Glutamine can directly feed the tricarboxylic acid (TCA) cycle or support glutathione (GSH) homeostasis (DeBerardinis and Cheng, 2010), which are known to be exquisitely intertwined (Chandel, 2015; O'Neill and Pearce, 2016). To elucidate the origin of the mitochondrial reprogramming in G/s1-deficient macrophage, metabolic pathway enrichment analysis was first performed in resting and reparative Mac ${ }^{\Delta \mathrm{Gls} 1} \mathrm{PCMs}$. This analysis highlighted down-regulation of oxidative phosphorylation and GSH synthesis KEGG pathways (i.e, NADP, folate and GSH metabolism) (Fig. S4a). Thus, to dissect the interplay between the mitochondrial metabolic repurposing and the perturbation in antioxidant metabolism in Mac ${ }^{\Delta \mathrm{Gls} 1}$ macrophages, we next performed topological analyses using CoMBI-T profiling (Jha et al., 2015) and compared predictions to DreamBio that allows direct computational KEGG pathway mapping. CoMBI-T and DreamBio profiling analyses featured a link between Mac ${ }^{\triangle \mathrm{Gls} 1} \mathrm{PCM}$ mitochondrial metabolic reprogramming and lower expression of tricarboxylic acid (TCA) cycle and aspartate-arginino-succinate (AAS) shunt genes. These genes are interconnected to metabolism of glutamine per se, carbohydrates, hexosamine and fatty acids (Fig. 4a and S4b). CoMBI-T predictions also revealed that the redox status of reparative Mac ${ }^{\Delta \mathrm{Gls} 1} \mathrm{PCMs}$ may connect to the one carbon cycle-centered module known to support the transsulfuration pathway (i.e, GSH synthesis) (Fig. 4a). KEGG mapping with DreamBio offered an alternative visualization of the perturbed transcriptomic pathways in reparative G/s1-deficient macrophages and highlighted additional reactions that were not yet predicted (Fig. S4b). For instance, in contrast to CoMBI-T, DreamBio analysis predicted downregulation of phosphogluconate dehydrogenase (PGD) and malic enzyme (ME1) in reparative Gls1-deficient macrophages. These are two key enzymes that contribute to GSH salvage pathway through NADPH generation to efficiently supply reduced glutathione and maintain macrophages redox status (Fig. S4b). Altogether, this unique approach helped pinpoint the metabolic origin on how Gls1-dependent glutamine metabolism can integrate canonical mitochondrial reprogramming (i.e, TCA and AAS shunt) and non-canonical redox status (i.e, GSH synthesis and GSH salvage pathway) to support macrophage reparative function.

Glutaminolysis bypasses the canonical route of mitochondrial glutamine catabolism to fuel OXPHOS and potentiate efferocytosis. We next validated predictions of topological analyses through functional testing. Although, there was no difference in $\alpha-K G$ carbon numbers that originated from ${ }^{13} \mathrm{C}$-glutamine in reparative Mac ${ }^{\Delta \mathrm{Gls} 1} \mathrm{BMDMs}$ (Fig. 3d), we first considered that the canonical glutaminolysis pathway, which relies on glutamate dehydrogenase (Glud1) to convert glutamate into $\alpha$-ketoglutarate $(\alpha-K G)$, would fuel the TCA cycle. However, we confirmed similar $\alpha-K G$ levels between controls and $\mathrm{Mac}^{\Delta \mathrm{Gls} 1} \mathrm{BMDMs}$ in a parallel metabolomic analysis we conducted (data not shown). Consistently, $\alpha-K G-d e p e n d e n t ~ K D M 6$ and teneleven translocation dioxygenase 2 (TET2) activities were similar between control and Mac ${ }^{\Delta \mathrm{Gls} 1}$ BMDMs (Fig. S4c). We also measured similar expression of KDM6 target genes such as Phf2, Phf8, Jarid2 or Mina (Fig. S4d) or global histone trimethylation modification in these cells (Fig. S4e). Treatment of control and $\mathrm{Mac}^{\Delta \mathrm{Gls} 1} \mathrm{BMDMs}$ with dimethyl $\alpha-K G$ did also not rescue the inhibitory effect of Gls1 deficiency on basal OCR (Fig. S4f). To genetically validate these findings, we generated macrophage deficient for Glud1 from mice lacking Glud1 in hematopoietic cells (Fig. 4b). In contrast to Gls1 deficiency, higher basal OCR and maximal respiration response (Fig. 4c), ATP production rate (Fig. 4d) and efferocytosis (Fig. 4e) were measured in reparative Glud1-deficient macrophages. These data prompted us to directly test the impact of Glud1-dependent canonical pathway in G/s1-deficient macrophages using epigallocatechin gallate (EGCG), a Glud1 inhibitor. Although this inhibition raised basal OCR and efferocytosis in reparative control macrophages, it was not sufficient to rescue G/s1deficient macrophage phenotypes (Fig. 4f). Altogether, these data suggest that G/s1-deficient macrophages metabolize less glutamate through a Glud1-independent non-canonical glutaminolysis pathway to limit the TCA cycle anapleurosis required for efficient efferocytosis.

Glutaminolysis-mediated GSH salvage but not synthesis potentiates efferocytosis. Based on our topological analyses, we next scrutinized the utilization of glutamate into the non-canonical 
GSH synthesis pathway that could prevent ATP leakage by limiting the $\gamma$-glutamyl futile cycle (Fig. 5a). A role of this futile cycle was unlikely as we observed similar amounts of the pyroglutamate intermediate (also known as PCA or 5-oxoproline) in Mac ${ }^{\Delta G \mid s 1}$ BMDMs (Fig. $\mathrm{S} 5 \mathrm{a}$ ). Treatment with $\mathrm{N}$-acetylcysteine (NAC) to replenish this futile cycle with cysteine, which is necessary for the glutamate pool to boost GSH synthesis (DeBerardinis and Cheng, 2010), had also no effect on OCR and efferocytosis in Mac ${ }^{\Delta \mathrm{Gls} 1}$ BMDMs (Fig. S5b). Nevertheless, the ratio between GSH (reduced glutathione)/GSSG (glutathione disulfide), known to scavenge reactive oxygen species (ROS) (DeBerardinis and Cheng, 2010), was greatly reduced in resting and reparative Mac ${ }^{\Delta \mathrm{Gls} 1} \mathrm{PCMs}$ (Fig. S5c). Although total glutathione concentration was decreased to some extent in reparative Mac ${ }^{\triangle \mathrm{Gls}}{ }^{1} \mathrm{PCMs}$, the decrease in the NADPH/NADP ratio was more strongly correlated to the GSH/GSSG ratio in resting and reparative Mac ${ }^{\Delta \mathrm{Gls} 1} \mathrm{PCMs}$ (Fig. S5c). These findings functionally validated findings from topological analyses and suggest a defect in GSH salvage pathway (i.e, NADPH-producing pathways to recycle GSSG into GSH) in Gls1deficient macrophages. To test whether this salvage pathway could contribute the high energy requirement of efferocytosis, we next restored this pathway by direct GSH supplementation (Fig. S5d). Unexpectedly, this treatment raised basal OCR levels, ATP production and efferocytosis in reparative Mac ${ }^{\Delta \mathrm{Gls} 1} \mathrm{BMDMs}$ to the levels of control macrophages (Fig. 5b). Thus, defective energy-intensive efferocytosis in G/s1-deficient macrophages is linked to perturbed GSH salvage pathway.

Glutaminolysis couples oxidative stress buffering to OXPHOS through non-canonical glutamine transamination metabolism to support the high energy demand of efferocytosis. We next reasoned that NADPH generation depend on a limited number of enzymes present in the mitochondria including isocitrate dehydrogenase or malic enzyme. Topological analysis suggested a role of the malate-pyruvate cycling pathway (i.e, reduced ME1 expression) in $\mathrm{Mac}^{\Delta \mathrm{Gls} 1} \mathrm{PCM}$ (Fig. S4b), that directly relies on the non-canonical transaminase-dependent malate-aspartate shuttle (Fig. 3b). This shuttle is also nested to the AAS shunt to maximize the number of ATP molecules produced in mitochondria (DeBerardinis and Cheng, 2010) (Fig. 5a). Thus, we next inhibited aspartate aminotransferases $\left(\mathrm{GOT}_{\mathrm{s}}\right)$-dependent transamination with aminooxyacetic acid (AOA) (Fig. 5a). Interestingly, AOA treatment reduced not only GSH levels (Fig. S5d) but also basal OCR and this limited efferocytosis in control macrophages to the levels of reparative $\mathrm{Mac}^{\Delta \mathrm{Gls} 1}$ BMDMs (Fig. 5c). Treatment with $A O A$ also inhibited efferocytosis in reparative Glud1-deficient BMDMs (Fig. 5d) or during continued clearance of $A C s$ in resolving control and Mac ${ }^{\Delta G \mid s 1} B M D M s$ (Fig. S5e). We validated these observations by showing that reduced expression of Got1 and Got2 by siRNA in BMDMs prevented the engulfment of apoptotic cells (Fig. S5f). Finally, we validated the role of non-canonical glutamine transamination pathway in the energy-intensive engulfment process by blocking the AAS shunt with AOA, which reduced $F$-actin immunostaining to the same level after AC engulfment in reparative control and $\mathrm{Mac}^{\Delta \mathrm{Gls} 1} \mathrm{BMDMs}$ (Fig. 5e). Thus, our results reveal that glutaminolysis and non-canonical transaminase pathways are preferentially used to maximize the number of ATP molecules produced in mitochondria to favor actin dynamics and maximize efferocytosis, which is linked to the ability of these pathways to power cells with the reducing equivalents necessary to detoxify ROS. 


\section{Discussion}

Macrophages require substantial amount of nutrients after ingestion of apoptotic cells (i.e, efferocytosis) to maintain normal tissue function (Han and Ravichandran, 2011). We and others have demonstrated that efferocytes can adapt their metabolism to face cholesterol and fatty acid overload during this process (A-Gonzalez et al., 2009)(Yvan-Charvet et al., 2010)(Viaud et al., 2018)(Zhang et al., 2019). Increased glucose uptake preceding AC engulfment could also prime continued AC clearance (Morioka et al., 2018). We now uncover that Gls1-dependent glutaminolysis is required to optimize AC clearance upon IL-4 stimulation or continued AC uptake by efficiently reprogramming macrophage metabolism. Indeed, conversion of glutamine through non-canonical transaminase pathways couples oxidative stress buffering to ATP production to meet the demand for high energy actin dyamics and cytoskeletal rearrangements.

Glutamine is considered a conditionally essential amino acid because of its role during metabolic stress, including injury (DeBerardinis and Cheng, 2010; O'Neill and Pearce, 2016). Glutamine utilization is increased in alternative macrophage polarization (Jha et al., 2015) and predicted macrophage activation profiles in aortas of atherosclerotic mouse models (Tavakoli et al., 2017). However, the fundamental link between Gls1 and efferocytosis in this setting remains to be elucidated. Strikingly, despite enhanced glutamine utilization in the aortas of atherosclerotic mice, we observed impaired glutamate conversion and reduced Gls1 expression, reflecting perturbed glutaminolysis. In our mouse and human datasets, we also observed that Gls1 expression within plaques was positively associated to canonical alternative polarization markers suggesting that glutaminolysis, rather than glutamine uptake could predict macrophage polarization. Gls1 expression also negatively correlated with necrotic cores.

Modulation of glutamine metabolism can lead to strikingly different phenotypes. For instance, it has recently been suggested that glutaminolysis could support $\alpha-K G$ generation to orchestrate the jumonji domain containing-3 (Jmjd3, KDM6B)-dependent epigenetic reprogramming of alternatively activated macrophages (Liu et al., 2017) or promote mTORC1 signaling during Th1 differentiation (Jonhson et al., 2018). In the current setting, we neither observed perturbations of $\alpha-K G$-dependent epigenetic reprogramming or upregulation of glycolysis and downstream mTOR signaling pathways in glutaminolysis deficient macrophages. Glutamine can be converted through glutamate dehydrogenase (Glud1) or non-canonically via transaminases. Unexpectedly, Glud1 deficiency exhibited an opposite efferocytosis phenotype relative to Gls1 deficiency. Indeed, high-throughput transcriptional and metabolic profiling revealed that macrophage resolving functions rely on a non-canonical transaminase pathway. The transaminase-dependent malate-aspartate shuttle is nested to the AAS shunt and requires glutamate to regenerate NADH, which is used to transfer electrons to the electron transport chain (ETC). This is required to maximize the number of ATP molecules produced in mitochondria and potentially favor NADPH production through ME-dependent malate-pyruvate cycling pathway to support GSH salvage pathway (DeBerardinis and Cheng, 2010). Consistently, all markers of these pathways were downregulated in Gls1-deficient macrophages. Although reduced GSH levels could explain the higher mitochondrial ROS in Gls1-deficient macrophages and mitochondrial redox status has been previously shown to metabolically program macrophage effector functions (Jais et al., 2014; Vats et al., 2006), we excluded a causal role of mitochondrial ROS in the metabolic reprogramming and defective efferocytosis of these cells using pharmacological mitochondrial ROS scavengers. Thus, treatment of Gls1-deficient macrophages with GSH most likely improved oxidative phosphorylation and efferocytosis by limiting NADPH consumption, which 'energy value' can be roughly estimated at between 2.5 and 3.5 ATP equivalents for purposes of comparing energy inputs and outputs of metabolic pathways (Stipanuk and Caudill, 2013). The central role of ATP produced within mitochondria after glutamate is channeled into the malateaspartate shuttle by aspartate aminotransferase (GOT)-dependent transamination in efferocytes was ultimately highlighted by directly targeting GOTs, mitochondrial complex II and III or mitochondrial ATP transport. 
The metabolism of other amino acids has recently emerged to control macrophage effector functions. For example, BC-AAs cause itaconate accumulation, a hallmark of macrophage proinflammatory response (Papathanassiu et al., 2017)(O'Neill and Artyomov 2019). The group led by Tabas et al. have also recently reported that the metabolism of ACderived arginine or ornithine to putrescine is required for continual efferocytosis by a mechanism involving cytoskeletal rearrangements (Yurdagul et al., 2020) and potentially linked to mitochondrial fission (Wang et al., 2017). This pathway is induced in alternatively activated macrophages (Jha et al., 2015), known to enhance efferocytic response for efficient tissue repair (Bosurgi et al., 2017). Impaired macrophage glutaminolysis likely occurs independently of the aforementioned amino acid pathways as similar levels of BC-AAs and putrescine were observed in Gls1-deficient macrophages. Furthermore, inhibition of BCAT or ornithine supplementation did not rescue the defective efferocytosis of these cells.

The high energy demand for cytoskeletal rearrangements during efferocytosis has previously been suggested but the underlying mechanisms remain poorly understood (Elliott and Ravichandran, 2016). Actin remodeling is an energy-intensive process that can be regulated by polymerization and depolymerization steps and formation of membrane ruffling for AC recognition and internalization (Han and Ravichandran, 2011). Rac1 and Cdc42 are key regulators of these processes. Along with reduced ATP production in Gls1-deficient macrophages, we observed both impaired actin polymerization and reduced Cdc42 and Rac1 activities. We found this mechanism was independent of the recently identified transcriptional regulation of Rac1 by GTP-exchange factor Dbl (Yurdagul et al., 2020). Thus, several complementary mechanisms exist to sustain an efficient successive clearance of ACs. We now identified for the first time that macrophage glutaminolysis supports the high energy demand for F-actin remodeling during this process.

Collectively, our results reveal a novel dependence on transaminases for metabolism of the glutamine carbon skeleton during key macrophage effector functions that are required for tissue repair. Mechanistically, these reactions orchestrate ATP generation through the ETC in the presence of IL-4 or during continued apoptotic cell clearance. These findings support the larger concept that any process that compromises the metabolic reprogramming and ATP production of efferocytes will likely result in dire pathologic consequences. Conversely, therapeutic interventions to enhance macrophage glutaminolysis and boost efferocytosis have the potential to subvert maladaptive inflammation and tissue necrosis.

Acknowledgments. We thank Béatrice Caraveo for computional development of DreamBio, a novel topological tool for Integrated Network Analysis. We thank Samantha Fernandez for the non-invasive study of atheroma plaques by ultrasound echography as part of the European Center for Research in Imaging (Cerimed), Dr Fréderic Labret for assistance with flow cytometry, Dr Véronique Corcelle for assistance in animal facilities and Dr. Marie Irondelle for assistance with confocal microscopy.

Financial support and sponsorship. This work was supported by grants from the Fondation de France (FDF) and the European Research Council (ERC) consolidator program (ERC2016COG724838) to L.Y.C. CCMA electron microscopy equipments have been funded by the Région Sud - Provence-Alpes-Côte d'Azur, the Conseil Départemental des Alpes Maritimes, and the GIS-IBiSA

Declaration of interests: The authors have declared that no conflict of interest exists. 


\section{Figure legends}

Figure 1: Macrophage-GIs1 deletion impairs efferocytosis in vitro and in vivo. (a) Western blot of Gls1 protein (left) and Glutamate levels (right) in Control or Mac ${ }^{\Delta G \mid s 1} \mathrm{PCMs}$. (b) Efferocytic index gating strategy (left) and quantification (right) measured by flow cytometry after 45min exposure with apoptotic cells (ACs) in BMDMs at steady state or after overnight IL-4 stimulation. Efferocytic index was calculated as follows: (number of macrophages with ACs/total number of macrophages) $\times 100$. (c) Efferocytic index gating strategy (Left) and quantification (right) measured by flow cytometry after one $(45 \mathrm{~min})$ or two $(45 \mathrm{~min}+1$-hour rest $+45 \mathrm{~min}$ ) incubations with ACs in control or Mac ${ }^{\Delta \mathrm{Gls} 1}$ BMDMs. (d) Efferocytic index gating strategy (left) and quantification (right) measured by flow cytometry in control or Mac ${ }^{\Delta \mathrm{GIs} 1}$ Kupffer cells (KC) and red pulp macrophages (RPM) after labelled apoptotic thymocytes i.v. injection._All values are mean \pm SEM and are representative of at least one experiment $(n=4-$ 6 independent animals). ${ }^{*} P<0.05$ compared to control.

Figure 2: Myeloid-GIs1 deletion impairs efferocytosis in the pathological process of atherosclerosis. (a) Representative sections (left) and quantification (right) of aortic plaques from $\mathrm{ApoE}^{-/-}$or ApoE ${ }^{-/-} \mathrm{Mac}^{\Delta \mathrm{Gls} 1}$ mice (12 weeks WD) stained for Oil Red O and Hematoxylin Eosin. Scale bar: $200 \mu \mathrm{m}$. (b) Oil red O stained descending aortas from $\mathrm{ApoE}^{-{ }^{--}}$or $\mathrm{ApoE}^{-/-}$ $\mathrm{Mac}^{\Delta \mathrm{Gls} 1}$ mice maintained on a WD for 12 weeks. (c) Echography (left) and quantification (right)

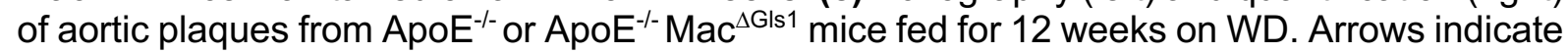
plaque areas. (d) Representative images (top) and quantification (bottom) of TUNEL ${ }^{+}$cells in aortic plaques from $\mathrm{ApoE}^{-/-}$or $\mathrm{ApoE}^{-l-} \mathrm{Mac}^{\Delta \mathrm{Gls} 1}$ mice (12 weeks WD). Green and blue arrow depict TUNEL negative nuclei and TUNEL-positive nuclei, respectively. (e) Correlation between Gls1 expression and human atherosclerotic plaque complexity in the Maastricht Pathology collection. All values are mean \pm SEM and are representative of at least one experiment $(\mathrm{n}=6-9) .{ }^{*} P<0.05$ compared to control.

Figure 3 Glutamine metabolism supports the high energy requirement of efferocytosis. (a) Quantification of $A C$ binding and internalization after treatment with $5 \mu \mathrm{M}$ cytochalasin $\mathrm{D}$ for 15 min before the addition of $A C$ in control or Mac ${ }^{\Delta G l s 1}$ BMDMs stimulated overnight +/- IL-4. (b) Transmission electron microscopy imaging of control or Mac ${ }^{\Delta \mathrm{Gls} 1} \mathrm{PCMs}$. Left scale bar: $10 \mu \mathrm{m}$. Right scale bar: $5 \mu \mathrm{m}$. (c) Representative images of control or Mac ${ }^{\Delta \mathrm{Gls} 1} \mathrm{BMDMs}$ stimulated overnight with IL-4 and stained for F-actin (Red) and ACs (green). Scale bar: 10 $\mathrm{mm}$. F-actin staining localized around the phagocytic cup was quantified. (d) Carbon fluxes using U-13C-glutamine. U-13C glutamine was added in the medium of non-stimulated and IL-4treated macrophages. Circle sizes are scaled with respect to pool size for individual metabolites in each condition. Thin black arrows represent known metabolic pathway connections; background arrows indicate deduced major metabolic flows in alternatively activated macrophages. (e) OCR measured by Seahorse after one incubation with ACs in control or Mac ${ }^{\Delta G \mathrm{Is} 1}$ BMDMs in basal conditions or (f) stimulated overnight with IL-4. (g) OCR measurement after two exposures with $\mathrm{ACs}$ in control or Mac ${ }^{\Delta \mathrm{Gls} 1} \mathrm{BMDMs}$. (h) Schematic representation of mitochondria electron transport chain and its pharmacological inhibitors. (i) OCR quantification and (j) efferocytic index of control or Mac ${ }^{\triangle G l s 1}$ BMDMs at steady state or after overnight IL-4 stimulation +/- Tempol, Mitoquinol, 3NPA or antimicyn A. All values are mean \pm SEM and are representative of at least one experiment $(n=3-9) .{ }^{*} P<0.05$ compared to control.

Figure 4: Macrophages utilize a non-canonical glutamine metabolism pathway to promote efficient efferocytosis. (a) CoMBI-T profiling analysis from RNAseq data of alternatively activated Gls1-deficient or sufficient PCMs. (b) Glud1 protein expression assessed by Western blotting in control or HSC ${ }^{\Delta G l u d 1}$ PCMs. (c) OCR and (d) ATP production rate measured by Seahorse in control or Mac ${ }^{\Delta \mathrm{Glud1}} \mathrm{BMDMs}$ at steady state or after overnight $\mathrm{IL}-4$ stimulation. (e) efferocytic index in control or Mac ${ }^{\Delta \text { Glud1 }}$ BMDMs at steady state or after overnight IL-4 stimulation. (f) OCR quantification (left) and efferocytic index (right) from control 
or Mac ${ }^{\Delta G l s 1}$ BMDMs in basal conditions or overnight IL-4 stimulation +/- EGCG. All values are mean \pm SEM and are representative of at least one experiment $(n=3-8)$.

${ }^{*} P<0.05,{ }^{* * *} P<0.0005$ compared to control.

Figure 5: Non-canonical transaminase pathway supports glutamine carbon skeleton metabolism to promote cytoskeletal rearrangement and corpse engulfment. (a) Schematic representation of glutamate incorporation into metabolic cycles. (b) OCR quantification (left), ATP production (middle) and efferocytic index (right) in control or Mac ${ }^{\Delta \mathrm{Gls} 1}$ BMDMs in basal conditions or overnight IL-4 stimulation +/- GSH. (c) OCR quantification (left) and efferocytic index (right) in control or Mac ${ }^{\Delta \mathrm{Gls} 1} \mathrm{BMDMs}$ in basal conditions or overnight IL-4 stimulation +/- AOA. (d) OCR quantification (left) and efferocytic index (right) in control or

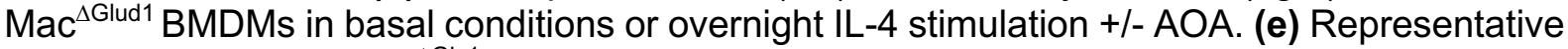
images of control or Mac ${ }^{\Delta \mathrm{Gls} 1} \mathrm{BMDMs}$ stimulated overnight with IL-4 in presence or absence of AOA and stained for F-actin (Red) and ACs (green). Scale bar: 10 $\mathrm{mm}$. F-actin staining localized around the phagocytic cup was quantified. All values are mean \pm SEM and are representative of at least one experiment $(\mathrm{n}=3-12) .{ }^{*} P<0.05$ compared to control. 
Material and Methods

KEY RESOURCES TABLE

\begin{tabular}{|c|c|c|}
\hline REAGENT or RESOURCE & SOURCE & IDENTIFIER \\
\hline \multicolumn{3}{|l|}{ Antibodies } \\
\hline Anti- $\alpha$-actine HRP & Santa Cruz & $\begin{array}{l}\text { SC-32251HRP } \\
\text { RRID:AB_262054 }\end{array}$ \\
\hline Anti-CD68 (clone FA-11) & Bio-Rad & $\begin{array}{l}\text { MCA1957 } \\
\text { RRID:AB_322219 }\end{array}$ \\
\hline Anti-Glud1 & Abcam & $\begin{array}{l}\text { ab166618 } \\
\text { RRID:AB_2815030. }\end{array}$ \\
\hline Anti-Glutaminase & Abcam & $\begin{array}{l}\text { ab93434 } \\
\text { RRID:AB_10561964 }\end{array}$ \\
\hline Anti-IL-4 (clone 11B11) & BioXcell & $\begin{array}{l}\text { BE0045 } \\
\text { RRID:AB_1107707 }\end{array}$ \\
\hline Anti-Ki67 PE (clone 16A8) & Biolegend & $\begin{array}{l}\text { 652403 } \\
\text { RRID:AB_2561524 }\end{array}$ \\
\hline Anti-Rat Alexa Fluor 488 & ThermoFisher & $\begin{array}{l}\text { A-11006 } \\
\text { RRID:AB_2534074 }\end{array}$ \\
\hline c-Myc PE (Clone D84C12) & Cell Signaling & $\begin{array}{l}\text { 14819S } \\
\text { RRID:AB_2798629 }\end{array}$ \\
\hline CD115 PE (clone AFS98) & eBioscience & $\begin{array}{l}\text { 12-1152-82 } \\
\text { RRID:AB_465808 }\end{array}$ \\
\hline CD11b Brilliant Violet 510 (cloneM1/70) & Biolegend & $\begin{array}{l}101263 \\
\text { RRID:AB_2629529 }\end{array}$ \\
\hline CD11c APC (clone N418) & BD Biosciences & $\begin{array}{l}117309 \\
\text { RRID:AB_313778 }\end{array}$ \\
\hline CD206 PerCp-Cy5.5 (clone C068C2) & Biolegend & $\begin{array}{l}141715 \\
\text { RRID:AB_2561991 }\end{array}$ \\
\hline CD301 FITC (clone ER-MP23) & Bio-Rad & $\begin{array}{l}\text { MCA2392 } \\
\text { RRID:AB_872014 }\end{array}$ \\
\hline CD45 APC-Cy7 (clone 30-F11) & BD Biosciences & $\begin{array}{l}557659 \\
\text { RRID:AB_396774 }\end{array}$ \\
\hline CD64 Brillant Violet 421 (clone X54-5/7.1) & Biolegend & $\begin{array}{l}\text { 139309 } \\
\text { RRID:AB_2562694 }\end{array}$ \\
\hline F4/80 PE-Cy7 (clone BM8) & Biolegend & $\begin{array}{l}123114 \\
\text { RRID:AB_893478 }\end{array}$ \\
\hline Histone H3 Alexa fluor 647 (clone D1H2) & Cell Signaling & $\begin{array}{l}\text { 12230S } \\
\text { RRID:AB_2797852 }\end{array}$ \\
\hline ICAM-2 alexa fluor 647 (clone 3C4(MIC2/4)) & Biolegend & $\begin{array}{l}105612 \\
\text { RRID:AB_2658040 }\end{array}$ \\
\hline MerTK PE (clone 2B10C42) & Biolegend & $\begin{array}{l}\text { 151506 } \\
\text { RRID:AB_2617037 }\end{array}$ \\
\hline PD-L2 APC (clone B7-DC) & Biolegend & $\begin{array}{l}107210 \\
\text { RRID:AB_2566345 }\end{array}$ \\
\hline Phalloidine Alexa Fuor 594 & InVitrogen & $\begin{array}{l}\text { A12381_ } \\
\text { RRID:AB_2315633 }\end{array}$ \\
\hline $\begin{array}{l}\text { Phospho-S6 Ribosomal Protein (ser235/236) PE (clone } \\
\text { D57.2.2E) }\end{array}$ & Cell Signaling & $\begin{array}{l}\text { 5316S } \\
\text { RRID:AB_10694989 }\end{array}$ \\
\hline $\begin{array}{l}\text { Tri-Methyl-Histone H3 Lys27 Alexa fluor } 647 \text { (clone } \\
\text { C36B11) }\end{array}$ & Cell Signaling & $\begin{array}{l}\text { 12158S } \\
\text { RRID:AB_2797834+ } \\
\text { E:F }\end{array}$ \\
\hline $\begin{array}{l}\text { Tri-Methyl-Histone H3 Lys4 Alexa fluor } 647 \text { (clone } \\
\text { C42D8) }\end{array}$ & Cell Signaling & $\begin{array}{l}\text { 12064S } \\
\text { RRID:AB_2797813 }\end{array}$ \\
\hline \multicolumn{3}{|l|}{ Chemicals, Peptides, and Recombinant Proteins } \\
\hline${ }^{13} \mathrm{C}$ glutamine & Sigma & 605166 \\
\hline${ }^{14} \mathrm{C}$ glutamine & PerkinElmer & NEC451050UC \\
\hline
\end{tabular}




\begin{tabular}{|c|c|c|}
\hline${ }^{3} \mathrm{H}$ thymidine & PerkinElmer & NET027E250UC \\
\hline $3 N P A$ & Sigma & 164603 \\
\hline aKG & Sigma & 349631 \\
\hline Annexin V PE & Biolegend & 640908 \\
\hline Antimycin & Sigma & A8674 \\
\hline $\mathrm{AOA}$ & Sigma & C13408 \\
\hline Aspartate & Sigma & MAK095 \\
\hline Azacytidine & Sigma & A2385 \\
\hline BCAT inhibitor & Cayman Chemical & 9002002 \\
\hline Bovine serum Albumin (BSA) & Sigma & A7030 \\
\hline BPTES & Sigma & SML0601 \\
\hline Cell Trace Violet proliferation kit & ThermoFisher & C34557 \\
\hline CFSE & ThermoFisher & C34570 \\
\hline Collagenase D & Sigma & 11088882001 \\
\hline DAPI & Sigma & D9542 \\
\hline Substrate HRP & Sigma & WBKLS0500 \\
\hline EGCG & Sigma & E4143 \\
\hline Eosin & DiaPath & C0363 \\
\hline FCCP & Sigma & C2920 \\
\hline Fetal bovine serum & Fisher Scientific & 12350273 \\
\hline Gabapentin & Sigma & G154 \\
\hline$\overline{\mathrm{GSH}}$ & Sigma & G4251 \\
\hline GSK-J4 & Sigma & SML0701 \\
\hline HBSS & Fisher Scientific & 14175053 \\
\hline Hematoxylin Solution & Sigma & HHS32 \\
\hline High-Capacity cDNA reverse transcription kit & Applied Biosystems & 4368814 \\
\hline $\mathrm{IL}-4$ & Peprotech & $200-04$ \\
\hline L-Glutamine & ThermoFisher & 25030024 \\
\hline L-ornithine & Sigma & 75440 \\
\hline Lipofectamine RNAiMAX & Fisher Scientific & 13778150 \\
\hline Lysing buffer & BD Biosciences & 555899 \\
\hline M-CSF & Miltenyi & $130-101-704$ \\
\hline Mitoquinol & Cayman Chemical & 89950 \\
\hline MitoSOX Red & Thermofisher & M36008 \\
\hline Mouse Foxp3 Buffer Set & Biosciences & 560409 \\
\hline NAC & Sigma & A7250 \\
\hline$\overline{\mathrm{OCT}}$ & TEK & $4583-01$ \\
\hline Oil Red O & Sigma & 00625 \\
\hline Oligomycin & Sigma & 75351 \\
\hline PBS 1X & Fisher Scientific & 14190169 \\
\hline Penicillin-Streptomycin & Fisher Scientific & 15140130 \\
\hline Percoll & Sigma & P1644 \\
\hline PFA 4\% & VWR International & 9713.1000 \\
\hline Poly-I:C & Invivogen & TLRL-PIC-5 \\
\hline Protease inhibitor cocktail & Sigma & P8340 \\
\hline RIPA buffer & Cell signaling & 9806 \\
\hline RNeasy Plus Mini Kit (250) & QIAGEN & 74136 \\
\hline ROS & Thermofisher & C6827 \\
\hline
\end{tabular}




\begin{tabular}{|c|c|c|}
\hline Rotenone & Sigma & R8875 \\
\hline RPMI medium & Fisher Scientific & 31870074 \\
\hline Tempol & Sigma & 581500 \\
\hline \multicolumn{3}{|l|}{ Critical Commercial Assays } \\
\hline DNA Hydroxymethylation (5-hmC) ELISA & Euromedex & $\mathrm{P}-1032-96$ \\
\hline Glutamine/Glutamate-Glo ${ }^{\mathrm{TM}}$ Assay & Promega & J8022 \\
\hline GSH/GSSG-Glo TM Assay & Promega & V6611 \\
\hline KDM6A/ KDM6B Activity & Abcam & ab156910 \\
\hline LabAssay(TM) Cholesterol & Sobioda & W1W294-65801 \\
\hline Rac1 G-LISA & Euromedex & CS-BK128 \\
\hline Triglycerides assay & Diasys & 157109910021 \\
\hline TUNEL Assay Kit - FITC & Abcam & $a b 66108$ \\
\hline \multicolumn{3}{|l|}{ Deposited Data } \\
\hline RNAseq & NCBI GEO & $\begin{array}{l}\text { GEO: GSE to } \\
\text { complete }\end{array}$ \\
\hline \multicolumn{3}{|l|}{ Experimental Models: Organisms/Strains } \\
\hline Mouse: ApoE KO & Jackson Laboratory & $\begin{array}{l}002052 \\
\text { RRID:IMSR_JAX:00 } \\
2052\end{array}$ \\
\hline Mouse: Gls1fl/fl & $\begin{array}{l}\text { Pr. Stephen Rayport's } \\
\text { lab }\end{array}$ & N.A. \\
\hline Mouse: Glud1fl/fl & $\begin{array}{l}\text { Pr. Pierre Maechler's } \\
\text { lab }\end{array}$ & N.A. \\
\hline Mouse: LyzMcre & Jackson Laboratory & $\begin{array}{l}004781 \\
\text { RRID:IMSR_JAX:00 } \\
4781\end{array}$ \\
\hline Mouse: $\mathrm{Mx} 1^{\text {cre }}$ & Jackson Laboratory & $\begin{array}{l}003556 \\
\text { RRID:IMSR_JAX:00 } \\
3556\end{array}$ \\
\hline \multicolumn{3}{|l|}{ Oligonucleotides } \\
\hline Got1 siRNA & Dharmacon & L-043492-01-0010 \\
\hline Got2 siRNA & Dharmacon & L-043495-01-0010 \\
\hline Scramble siRNA & Dharmacon & D-001810-01-05 \\
\hline \multicolumn{3}{|l|}{ Software and Algorithms } \\
\hline FlowJo & Tree Star & N.A. \\
\hline ImageJ & $\mathrm{NIH}$ & N.A. \\
\hline Phantasus & $\begin{array}{l}\text { http://genome.ifmo.ru/ } \\
\text { phantasus/ }\end{array}$ & N.A. \\
\hline Prism8 & GraphPad & N.A. \\
\hline StepOne Software v.2.2.2 & Applied Biosystem & N.A. \\
\hline \multicolumn{3}{|l|}{ Other } \\
\hline Western Diet & Ssniff & TD88137 \\
\hline
\end{tabular}

Human atherosclerosis. Tissue collection was part of the Maastricht Pathology Tissue Collection (MPTC) and further storage and use of the tissue was in line with the Dutch Code for Proper Secondary use of Human Tissue and the local Medical Ethical Committee (protocol number 16-4-181).

Carotid arteries were collected from symptomatic patients undergoing carotid endarterectomy (CEA). Formalin-fixed, paraffin embedded (FFPE) $5 \mathrm{~mm}$-segments were alternated with frozen segments for RNA isolation. FFPE segments were then classified as fibrous cap atheroma with or without intraplaque hemorrhage (stable or unstable respectively) according to haematoxylin-eosin (HE) staining.1 Forty-four paired stable and unstable snap-frozen 
segments from 22 symptomatic patients undergoing CEA in the Maastricht human plaque study (MaasHPS) were only used for further microarray analysis when both adjacent plaque segments showed the same histological classification. Snap-frozen segments were pulverized and $5-20 \mathrm{mg}$ of material was subjected for transcriptomics. RNA isolation was performed by Guanidium Thiocyanate extraction and further purified with the Nucleospin RNA II kit (Macherey-Nagel GmbH\&Co). RNA quality and integrity were determined using the Agilent 2100 Bioanalyzer. Biotinylated cRNA was prepared with Illumina TotalPrep RNA Amplification Kit (Ambion, TX, U.S.A) and 750ng cRNA per sample was used for hybridization (Illumina Human Sentrix-8 V2.0, Beadchipß). Scanning was performed on the Illumina Beadstation 500 (Illumina, CA, U.S.A) and image analysis was done using the Illumina Beadstudio v3 Gene expression software. A total of 22,184 human transcripts were analyzed in R Bioconductor lumi package.2 Firstly, a variance stabilizing transformation, which is incorporated in the lumi package, was performed. Secondly, Robust Spline Normalization (RSN) algorithm in lumi package was applied to normalize the data. Differential gene expression analysis was done by using the function ImFit() provided in Limma R package on preprocessed transcriptomics data. 3

The 88 adjacent tissue sections were phenotyped extensively for plaque size, necrosis, inflammation (CD68, CD3, arginase, iNOS), SMCs (aSMA), collagen (Sirius red) and angiogenesis (CD31+ microvessel density, newly formed CD105+ microvessels, aSMA+ mature microvessels, Lyve+ lymphatic density). Pearson correlation analysis was performed to assess the association between gene expression and plaque phenotypical traits.

Mice. Gls $1^{\mathrm{fl} / \mathrm{fl}}$ mice were kindly provided by $\operatorname{Pr}$. Stephen Rayport and have been crossed to Lyz2 ${ }^{\text {Cre }}$ mice (B6.129P2-Lyz2tm1(cre)lfo/J, The Jackson Laboratory) or Mx1 $1^{\text {cre }}$ mice (B6.Cg$\mathrm{Tg}(\mathrm{Mx1}$-cre)1Cgn/J) and brought on ApoE-deficient genetic background (B6.129P2ApoEtm1Unc/J). Mx1 ${ }^{\text {cre }}$ mice (B6.Cg-Tg(Mx1-cre)1Cgn/J) were also crossed to Glud1 floxed mice (kindly provided by Pr. Pierre Maechler). For each experiment, co-housed littermate controls were used. Animal protocols were approved by the Institutional Animal Care and Use Committee of the French Ministry of Higher Education and Research and the Mediterranean Center of Molecular Medicine (Inserm U1065) and were undertaken in accordance with the European Guidelines for Care and Use of Experimental Animals. Animals had free access to food and water and were housed in a controlled environment with a 12-hour light-dark cycle and constant temperature $\left(22^{\circ} \mathrm{C}\right)$. Hyperlipidemia was induced by feeding the mice with a Western diet (TD88137, Ssniff) for 12 weeks. Mice were weighted every two weeks following Western diet induction.

Poly(l:C) induction. $M \times 1^{\text {cre }}$ mice were i.p. injected with $1 \mathrm{mg} / \mathrm{mL}$ poly $(\mathrm{l}: \mathrm{C})$ three times every two days(Kuhn et al., 1995). Mice were then used for experimentation 3 weeks later.

IL-4c in vivo treatment. Mice were i.p. injected with IL-4 complexed to anti-IL-4 mAb (IL-4c; containing $5 \mu \mathrm{g}$ of IL-4, PeproTech, and $25 \mu \mathrm{g}$ of anti-IL-4 clone 11B11, BioXcell). 36 hours later, PCMs were collected and analyzed by flow cytometry.

Cell culture, LPMs and BMDMs generation. BM cells were harvested from mouse femur and tibia and differentiated in the presence of recombinant mouse M-CSF (20 ng/ml; Miltenyi) in complete RPMI 1640 medium (Corning) containing 10mM glucose, $2 \mathrm{mM} \mathrm{L-glutamine,} 100 \mathrm{U} / \mathrm{ml}$ of penicillin/streptomycin, and $10 \%$ FBS for 7 days at $37^{\circ} \mathrm{C}$ and $5 \% \mathrm{CO} 2$.

Cell treatments. At day 7 BMDMs were collected, plated and activated overnight as indicated. Peritoneal cells were obtained by peritoneal lavage with $5 \mathrm{ml}$ of PBS. Cells were plated and cultured overnight in complete RPMI 1640 medium (Corning) containing $10 \mathrm{mM}$ glucose, $2 \mathrm{mM}$ L-glutamine, $100 \mathrm{U} / \mathrm{ml}$ penicillin/streptomycin, and $10 \% \mathrm{FBS}$ at $37^{\circ} \mathrm{C}$ and $5 \% \mathrm{CO}$. Cell were incubated overnight with the following treatments: IL-4 (20ng/mL, Peprotech), AOA $(200 \mu \mathrm{M}$, Sigma), Dimethyl-a-ketoglutarate (1mM, Sigma), EGCG $(100 \mu \mathrm{M}$, Sigma), GSH (10mM, Sigma), cytochalasin D ( $5 \mu \mathrm{M}$, Sigma), L-ornithine (1mM, Sigma), BCATc inhibitor $(20 \mu \mathrm{M}$, 
Cayman Chemical), Gabapentin $(10 \mu \mathrm{g} / \mathrm{mL}$, Sigma), 3NPA ( $1.68 \mathrm{mM}$, Sigma), Antimycin A

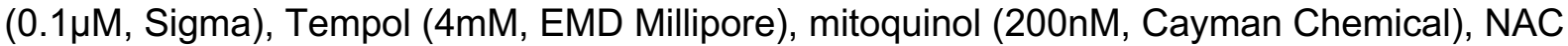
$(10 \mathrm{mM}$, Sigma). For glutamine and glucose deprivation, glucose or glutamine free media were used, and cells were deprived for 4 hours before the experiments.

siRNA transfection. Cells were transfected with Got1/Got2 siRNA (L-043492-01-0005 and L043495-01-0005, Dharmacon) or control siRNA (D-001810-01-05, Dharmacon) (referred to as Scbl) at $30 \mathrm{nM}$ using Lipofectamine RNAiMAX (Life Technologies), according to the manufacturer's instructions.

Lentivirus overexpression. Cells were spin-transfected for $90 \mathrm{~min}$ at $2500 \mathrm{rpm}$ with Gls1, lentivirus (LVM(VB200119-1197bpk)-C from Vectorbuilder) or control lentivirus (LVM(VB200120-1215tyv)-C, Vectorbuilder) (referred to as Scbl) and used at MOI 10. After 6 hours, cells were washed, and the media replaced with fresh media.

Western Blotting. LPMs were cell-sorted and then lysed in RIPA buffer containing protease inhibitors cocktail (ThermoFisher) and agitated for 1 hour at $4^{\circ} \mathrm{C}$ before centrifugation at 14000 rpm for $10 \mathrm{~min}$ at $4^{\circ} \mathrm{C}$. Supernatants were sampled and later used for SDS-PAGE. Protein content was evaluated using Pierce ${ }^{\mathrm{TM}} \mathrm{BCA}$ assay kit (ThermoFisher). Protein samples were resolved on $10 \%$ SDS-PAGE gels and were then transferred onto polyvinylidene difluoride membrane using a wet transfer system. Membranes were blocked in $5 \%(\mathrm{w} / \mathrm{v})$ BSA in Trisbuffered saline-Tween for one hour at room temperature. Membranes were then incubated with primary antibody (anti-Glud1 or anti-Gls1 antibodies (Abcam)) followed by the appropriate horseradish peroxidase-conjugated secondary antibody. Anti $\alpha$-actin mAb (Santa Cruz) was used as loading control. Proteins were detected by substrate HRP (Sigma).

Analysis of atherosclerotic plaque. Mice were sacrificed and slowly perfused with $10 \mathrm{ml}$ of ice-cold PBS. The hearts and aortas were carefully excised and fixed in $4 \%$ paraformaldehyde containing $30 \%$ sucrose. The aortas were stained with Oil Red O (Sigma- O0625) to evaluate plaque neutral lipid content as previously described (Yun et al., 2017). The hearts were embedded in OCT compound (Gentaur) and stored at $-80^{\circ} \mathrm{C}$ before analysis. $10 \mu \mathrm{m}$ cryosections of the aortic sinus were prepared. Oil Red $\mathrm{O}$ staining was used to detect neutral lipid content in the plaque combined with a haematoxylin/eosin staining to analyse tissue architecture. Plaque macrophages were visualized using purified anti-CD68 mAb (clone FA11, AbD Serotec). Anti-rat Alexa Fluor 488-conjugated antibody (A-11006, Life technologies) was used for detection of CD68 staining. For analysis of plaque macrophage proliferation, antiKi67 PE conjugated mAb (clone 16A8, BioLegend) was used. Nuclei were revealed with DAPI counterstaining $(2 \mu \mathrm{g} / \mathrm{ml})$. TUNEL staining was performed using the DeadEnd ${ }^{\mathrm{TM}}$ Fluorometric TUNEL System (Promega). Plaque area quantification were measured with ImageJ software.

Echography. Animals were fully anesthetized with 1.5\% Isoflurane before and during ultrasound scanning. Before all ultrasound scanning, the hair of the mouse chest wall was carefully removed, and warm ultrasound transmission gel was liberally applied to ensure optimal image quality. Echocardiography was performed using a high-frequency Vevo2100 (Visualsonics)-imaging.

Transmission Electronic Microscopy. Cells were observed with transmission electron microscopy (TEM) for ultrastructural analysis. Cells were fixed in a $1.6 \%$ glutataraldehyde solution in $0.1 \mathrm{M}$ sodium phosphate buffer at room temperature (RT) and stored overnight at $4^{\circ} \mathrm{C}$. After three rinsing in $0.1 \mathrm{M}$ cacodylate buffer (15 min each), cells were postfixed in a $1 \%$ osmium tetroxide and $1 \%$ potassium ferrocyanide solution in $0.1 \mathrm{M}$ cacodylate buffer for 1 hour at RT. Cells were subsequently dehydrated in a series of acetone baths $(90 \%, 100 \%$ three times, 15 min each) and progressively embedded in Epon 812 resin (acetone / resin 1:1, $100 \%$ resin two times, 2 hours for each bath). Resin blocs were finally left to harden in a 60 ${ }^{\circ} \mathrm{C}$ oven for 2 days. Ultrathin sections $(70 \mathrm{~nm})$ were obtained with a Reichert Ultracut $\mathrm{S}$ 
ultramicrotome equipped with a Drukker International diamond knife and collected on 200 mesh copper grids. Sections were stained with lead citrate and uranyl acetate. TEM observations were performed with a JEOL JEM-1400 transmission electron microscope, equipped with a Morada camera, at a $100 \mathrm{kV}$ acceleration voltage.

In vitro efferocytosis analysis. BMDMs and PCMs were generated and stimulated as described above.

To generate apoptotic cells (ACs), thymii from C57BL/6J mice were harvested and mechanistically dissociated, filtered on $100 \mu \mathrm{m}$ nylons (Falcon), pelleted and resuspended in RPMI medium supplemented with $10 \%$ FBS. Apoptosis was induced by UV exposure at $312 \mathrm{~nm}$ for $10 \mathrm{~min}$ and cells were maintained in culture for an additional 2 hours. This method results in $70-90 \%$ apoptotis (Yvan-Charvet et al., 2010). ACs were labelled with CellTrace ${ }^{\mathrm{TM}}$ Violet Cell Proliferation kit (ThermoFisher) according to the manufacturer's instructions. Fluorescent ACs were washed twice with PBS before use.

For one round efferocytosis: Stained apoptotic cells (ACs) were added at a 5:1 ratio on plated macrophages for $45 \mathrm{~min}$.

For two rounds efferocytosis: Unlabelled apoptotic lymphocytes (ACs) were added at a 5:1 ratio on plated macrophages for $45 \mathrm{~min}$. Cells were then washed 3 times and macrophages were incubated for $1 \mathrm{~h}$. Stained apoptotic lymphocytes (ACs) were then added at a 5:1 ratio on macrophages for $45 \mathrm{~min}$.

Cells were washed 3 times and macrophages were stained and analysed for AC content and activation markers by flow cytometry. For Seahorse extracellular flux analysis ACs were injected directly, before drug treatment, during the assay.

Histology. BMDMs and ACs were generated and stimulated as described above. ACs were stained with CellTrace ${ }^{\text {TM }}$ CFSE (Invitrogen) for $30 \mathrm{~min}$ prior to one round efferocytosis. After $15 \mathrm{~min}$ of efferocytosis, BMDMs were washed with a $37^{\circ} \mathrm{C}$ heated medium and fixed for $10 \mathrm{~min}$ at $37^{\circ} \mathrm{C}$ with $4 \%$ paraformaldehyde culture medium. Cells were then saturated for $30 \mathrm{~min}$ in PBS $10 \%$ FBS. Cells were incubated for $30 \mathrm{~min}$ with Texas Red ${ }^{\mathrm{TM}}-\mathrm{X}$ Phalloidin (Invitrogen). Cells were then washed thoroughly, and coverslips were mounted with aqueous glue. Sections were observed the following day by fluorescent microscopy.

In vivo efferocytosis analysis. ACs were generated as described above. Mice were i.v injected with $2 * 10^{7}$ stained ACs and 1 hour later, the spleen and liver were collected and analysed by flow cytometry.

In vitro flow cytometry analysis. Cells were stained for 25 min at $4^{\circ} \mathrm{C}$ protected from light. For flow cytometry analysis the following list of antibodies was used: MitoSOX ${ }^{\mathrm{TM}}$ Red (ThermoFisher), ROS (Thermofisher, CM-H2DCFDA), CD206 PerCp-Cy5.5 conjugated (clone C068C2, BioLegend), PD-L2 APC conjugated (Clone B7-DC, Biolegend), MerTK PE conjugated (clone 2B10C42, BioLegend), CD115 PE conjugated (clone AFS98, eBioscience), CD64 Brillant Violet 421 conjugated (clone X54-5/7.1, BioLegend), F4/80 Pe-Cy7 conjugated (clone BM8, BioLegend), Annexin V (Biolegend), CD4 FITC conjugated (Clone RM4.5), CD8b FITC conjugated (Clone YTS156.7.7). Cells were then washed, centrifuged and data were acquired on BDFACSCanto flow cytometer. Analysis was performed using FlowJo software (Tree Star).

Intracellular flow cytometry. For all intracellular stainings: Phospho-S6 Ribosomal Protein (Ser235/236) PE conjugated (clone D57.2.2E, Cell signaling), c-Myc PE Conjugate (Clone D84C12, Cell signaling), Tri-Methyl-Histone H3 (Lys27) Alexa Fluor® 647 Conjugated (Clone C36B11), Histone H3 (Alexa Fluor® 647 Conjugated (Clone D1H2), Tri-Methyl-Histone H3 (Lys4) Alexa Fluor® 647 conjugated (Clone C42D8), CD4 APC conjugated (Clone RM4.5), CD8b Alexa Fluor ${ }^{\circledR} 647$ Conjugated (Clone YTS156.7.7). Cells were removed from media, stained for surface, fixed, then stained for intracellular proteins using Foxp3 Transcription Factor Fixation/Permeabilization kit (BD biosciences). 
In vivo flow cytometry analysis. Cells were collected from spleen, peritoneal cavity, bone marrow, liver and brain. Splenocytes were extracted by pressing spleens through a stainlesssteel grid. Peritoneal and bone marrow leukocytes were harvested by PBS lavage. Liver and brain were cut in small piece and digested for $30 \mathrm{~min}$ with HBSS medium containing $1,5 \mathrm{mg} / \mathrm{mL}$ collagenase $\mathrm{D}$ (Roche) at $37^{\circ} \mathrm{C}$. For liver and microglia preparation, an additional purification step was performed by Percoll gradient. Single-cell suspension was submitted to red blood cell lysis, filtration and centrifugation for $5 \mathrm{~min}$ at $1,500 \mathrm{rpm}$. Cell suspensions were stained with the appropriate antibodies for $30 \mathrm{~min}$ on ice protected from light. The following antibodies were used for macrophage flow cytometric analysis: CD11b Brillant Violet 510 conjugated (clone M1/70, BioLegend), CD115 PE conjugated (clone AFS98, eBioscience), CD45 APC-Cy7 conjugated (clone 30-F11, BD Biosciences), CD64 Brillant Violet 421 conjugated (clone X545/7.1, BioLegend), CD11C APC conjugated (clone N418, BioLegend), F4/80 Pe-Cy7 conjugated (clone BM8, BioLegend), CD206 PerCp-Cy5.5 conjugated (clone C068C2, BioLegend), CD301 FITC conjugated (clone ER-MP23, Bio-Rad). Cells were then washed, centrifuged and data were acquired on BDFACSCanto flow cytometer. Analysis was performed using FlowJo software (Tree Star).

Seahorse extracellular flux analysis. For extracellular flux assay, $1 \times 10^{5}$ BMDMs or LPMs were plated in a Seahorse Bioscience culture plate. Cells were then incubated overnight with different drugs and metabolites. OCR and ECAR was measured by an XF96 Seahorse Extracellular Flux Analyzer following the manufacturer's instruction. In the seahorse assay, cells were treated with oligomycin $(1 \mu \mathrm{M})$, FCCP $(1.5 \mu \mathrm{M})$, rotenone $(1 \mu \mathrm{M})$ and antimycin $\mathrm{A}$ $(0.1 \mu \mathrm{M})$. Each condition was performed in 3 replicates.

RNAseq. PCMs were obtained by lavage as described above. Then cells were stained with CD64 Brilliant Violet 421 conjugated (clone X54-5/7.1), ICAM-2 alexa fluor 647 conjugated (clone 3C4(MIC2/4)) and CD115 PE conjugated (clone AFS98). These antibodies allow us to separate the two subsets of peritoneal macrophages with only the major one expressing ICAM2 (Gautier et al., 2012; Kim et al., 2016). Cells were cell sorted on BD FacsAria flow cytometer. Total RNA was extracted with RNeasy Mini Kit (Qiagen) according to the manufacturer's protocol and quality was assessed by Nanodrop (Ozyme). Library construction were conducted as described previously (Jha et al., 2015). Libraries were sequenced at the Centre for Applied Genomics (SickKids, Toronto) using a HiSeq 2500 (Illumina).

Integrated network analyses. Network-based integration of metabolite and gene expression datasets was conducted using Shiny Gam as previously described (Jha et al., 2015). We also developed DreamBio, a complementary topological tool for Integrated Network Analysis mapped into KEGG pathway. The same strategy than Shiny Gam was used by downloading KEGG REACTION, KEGG ENZYME, KEGG COMPOUND, and KEGG GLYCAN databases (August 2013 version) except that GEPHI GEXF (graph exchange format) was converted from KGML to be analyzed through Sigma library js dedicated to graph drawing. Up and down regulated metabolic genes based on $p$ values calculated with Phantasus (Jha et al., 2015) were mapped into models maintaining all essential KEGG pathway attributes. DreamBio will become soon freely available at http://dreamsession.com/biotest/bioinfo/index.php?w=sigma.

Fluxomics. Metabolite extraction of BMDMs was performed on 2.5 million cells per well using $70^{\circ} \mathrm{C}$ aqueous $70 \%$ ethanol as described previously (Devos et al., 2019). At collection, cells were placed immediately on ice, the media was removed, and cells were washed three times with ice-cold PBS to remove residual media. Intracellular metabolites were extracted twice with hot ethanol. For LCMS, samples where dried under nitrogen flow and reconstituted in a milliQ water/acetonitrile (1:1) mixture for injection using a UPLC Acquity (Waters) separation system coupled with a Xevo G2 ToF (Waters). Compounds were ionized using an electrospray ionization source in negative mode. Data processing was performed in MATLAB (Mathworks, Inc.) using a custom-made in-house protocol. Compound identification was performed using 
both retention time of authentic standards and accurate mass with an accepted deviation of $0.005 \mathrm{Da}$. Raw data was converted to netCDF format using Chemstation (Agilent), before processing in MATLAB R2014b (Mathworks, Inc.) using PARADISe software. All MS sample processing and analysis were performed by MS-Omics, Inc. (Copenhagen, Denmark).

Metabolomics. Metabolomics analyses were performed at CriBioM as previously described (Aidoud et al., 2018). Briefly, control and Gls1-deficient BMDMs were treated with or without $20 \mathrm{ng} / \mathrm{mL} \mathrm{IL}-4$ as indicated in the figure legends. Metabolites were extracted by exposing cells to cold methanol and analyzed by LC-MS.

Typhoon ${ }^{T M}$ Biomolecular Imager. $2 \mu \mathrm{Ci}$ of $\left[{ }^{14} \mathrm{C}\right]$-labelled glutamine were i.v. injected and mice were sacrificed 15 minutes later. Aortas were harvested and the associated adipose tissue was carefully dissected and removed. Imaging for $\left[{ }^{14} \mathrm{C}\right]$-labelled glutamine was performed on Typhoon ${ }^{\mathrm{TM}}$ Biomolecular Imager (Amersham). Whole mount staining with Oil Red O paralleled this analysis on the same samples.

Thin-Layer Chromatography (TLC). Aortic tissues were homogenized with $5 \% \mathrm{HClO} 4$ solution and the radioactivity incorporated in this extract was measured before being dropped on silica-gel POLYGRAM precoated TLC sheets (Sigma). Separation of ${ }^{14} \mathrm{C}$ glutamine and the ${ }^{14} \mathrm{C}$ glutamine-derived glutamate was achieved in hexane/diethylether/formic acid (80:20:1 $\mathrm{v} / \mathrm{v} / \mathrm{v}$ ) running buffer. The radioactivity was quantified and expressed as a percentage of ingested radioactivity.

$\left.\Gamma^{4} \mathrm{C}\right\}$ Glutamine incorporation. BMDMs were generated and stimulated as described above. Four hours before the experiment, cells were deprived in glutamine or put in presence of medium containing $1 \mathrm{mM}$ glutamine. $\left[{ }^{14} \mathrm{C}\right]$-Glutamine $(1 \mu \mathrm{Ci})$ was added on cells for 18 hours. Cells were collected, centrifuged, washed with PBS, and resuspended in $\mathrm{NaOH}(0.1 \mathrm{~N}) / \mathrm{SDS}$ $(0,1 \%)$ before adding scintillation.

[ $\mathrm{H}$-Thymidine incorporation. BMDMs were generated and stimulated as described above. The day before the experiment, cells were stimulated overnight with IL-4 $(20 \mathrm{ng} / \mu \mathrm{L})$. $\left[{ }^{3} \mathrm{H}\right]-$ Thymidine $(1 \mu \mathrm{Ci})$ was added on cells in regular media for 2 hours. Cells were collected, centrifuged, washed with PBS, and resuspended in $\mathrm{NaOH}(0.1 \mathrm{~N}) / \mathrm{SDS}(0,1 \%)$ before adding scintillation.

Glutamine and glutamate measurement. To analyse macrophage glutamine and glutamate content we used a commercially available kit Glutamine/Glutamate-Glo ${ }^{\mathrm{TM}}$ Assay (Promega) in accordance with the manufacturer's instructions. Briefly, BMDMs and PCMs were incubated as previously described and washed three times with PBS to remove remaining cell culture medium. Cells were then subjected to osmotic lysis with DI water before following the manufacturer's instructions.

Plasma cholesterol and triglycerides content. Plasma cholesterol and triglyceride content was measured with LabAssayTM Cholesterol (Sobioda) and Triglycerides Reagent (Diasys) according to the manufacturer's protocol.

Intracellular GSH/GSSG assay. The reduced glutathione (GSH)/ oxidized glutathione (GSSG) balance was determined using GSH/GSSG-Glo ${ }^{\mathrm{TM}}$ Assay (Promega) according to the manufacturer's instructions.

Rac1 and Cdc42 activity assay. Rac1 and Cdc42 activities were determined with Rac1 GLISA Activation Assay Kit (Cytoskeleton BK128) and Cdc42 G-LISA Activation Assay Kit (Cytoskeleton BK127) according to the manufacturer's instructions. 
G-Actin/F-Actin assay. G-Actin/F-Actin activity was determined with G-Actin/F-Actin In Vivo Assay Biochem Kit (Cytoskeleton) according to the manufacturer's instructions.

Actin polymerization assay. Actin polarization activity was determined with Actin Polymerization Biochem Kit (Cytoskeleton) according to the manufacturer's instructions.

KDM6 activity assay. KDM6 activity was determined with KDM6A/ KDM6B Activity Quantification Assay Kit (Abcam) according to the manufacturer's instructions.

Tet2 activity assay. Tet2 activity was determined with MethylFlash Global DNA Hydroxymethylation (5-hmC) ELISA Easy Kit (Epigentek) according to the manufacturer's instructions.

Real-Time $q P C R$. Total RNA was isolated using the RNeasy Plus Mini kit (QIAGEN) and quantified using a Nanodrop (Ozyme). cDNA was prepared using $10 \mathrm{ng} / \mu \mathrm{l}$ total RNA by a RTPCR using a high capacity cDNA reverse transcription kit (Applied Biosystems), according to the manufacturer's instructions. Real-time qPCR was performed on cDNA using SYBR Green. qPCRs were performed on StepOne device from Applied Biosystem (France). Samples were performed in triplicates. Results are expressed in arbitrary units. Gls1 (GCACATTATTCACCCGGTAACC; CTGCCCACCCACCATCC, Thermofisher)

Statistics. Data are expressed as mean+/- SEM. Statistical analysis was performed using a 2-tailed $t$ test or ANOVA (with Tukey's post-test analysis) with GraphPad Prism software. A P value $\leq 0.05$ was considered as statistically significant. 


\section{References}

A-Gonzalez, N., Bensinger, S.J., Hong, C., Beceiro, S., Bradley, M.N., Zelcer, N., Deniz, J., Ramirez, C., Díaz, M., Gallardo, G., de Galarreta, C.R., Salazar, J., Lopez, F., Edwards, P., Parks, J., Andujar, M., Tontonoz, P., Castrillo, A. Apoptotic cells promote their own clearance and immune tolerance through activation of the nuclear receptor LXR. Immunity. 2009 Aug 21;31(2):245-58.

Aidoud, N., Delplanque, B., Baudry, C., Garcia, C., Moyon, A., Balasse, L., Guillet, B., Antona, C., Darmaun, D., Fraser, K., et al. (2018). A combination of lipidomics, MS imaging, and PET scan imaging reveals differences in cerebral activity in rat pups according to the lipid quality of infant formulas. FASEB J 32, 4776-4790.

Aspenström, P. (2004). Integration of signalling pathways regulated by small GTPases and calcium. Biochim Biophys Acta 1742, 51-8.

Bauer, T.M., Murphy, E. (2020). Role of Mitochondrial Calcium and the Permeability Transition Pore in Regulating Cell Death. Circ Res 126, 280-293.

Bosurgi, L., Cao, Y.G., Cabeza-Cabrerizo, M., Tucci, A., Hughes, L.D., Kong, Y., Weinstein, J.S., Licona-Limon, P., Schmid, E.T., Pelorosso, F., et al. (2017). Macrophage function in tissue repair and remodeling requires IL-4 or IL-13 with apoptotic cells. Science 356, $1072-$ 1076.

Caron, E., Hall, A. (1998). Identification of two distinct mechanisms of phagocytosis controlled by different Rho GTPases. Science 282, 1717-21.

Chandel, N.S. (2015). Evolution of Mitochondria as Signaling Organelles. Cell Metab 22, 204206.

DeBerardinis, R.J., and Cheng, T. (2010). Q's next: the diverse functions of glutamine in metabolism, cell biology and cancer. Oncogene 29, 313-324.

Devos, M., Mogilenko, D.A., Fleury, S., Gilbert, B., Becquart, C., Quemener, S., Dehondt, H., Tougaard, P., Staels, B., Bachert, C., et al. (2019). Keratinocyte Expression of A20/TNFAIP3 Controls Skin Inflammation Associated with Atopic Dermatitis and Psoriasis. J Invest Dermatol 139, 135-145.

Dixon, S.J., Lemberg, K.M., Lamprecht, M.R., Skouta, R., Zaitsev, E.M., Gleason, C.E., Patel, D.N., Bauer, A.J., Cantley, A.M., Yang, W.S., et al. (2012). Ferroptosis: an iron-dependent form of nonapoptotic cell death. Cell 149, 1060-1072.

Elliott, M.R., and Ravichandran, K.S. (2016). The Dynamics of Apoptotic Cell Clearance. Dev Cell 38, 147-160.

Fan, J., Ye, J., Kamphorst, J.J., Shlomi, T., Thompson, C.B., and Rabinowitz, J.D. (2014). Quantitative flux analysis reveals folate-dependent NADPH production. Nature 510, 298-302. Gautier, E.L., Shay, T., Miller, J., Greter, M., Jakubzick, C., Ivanov, S., Helft, J., Chow, A., Elpek, K.G., Gordonov, S., et al. (2012). Gene-expression profiles and transcriptional regulatory pathways that underlie the identity and diversity of mouse tissue macrophages. Nat Immunol 13, 1118-1128.

Grabner, R., Lotzer, K., Dopping, S., Hildner, M., Radke, D., Beer, M., Spanbroek, R., Lippert, B., Reardon, C.A., Getz, G.S., et al. (2009). Lymphotoxin beta receptor signaling promotes tertiary lymphoid organogenesis in the aorta adventitia of aged ApoE-/- mice. J Exp Med 206, 233-248.

Han, C.Z., and Ravichandran, K.S. (2011). Metabolic connections during apoptotic cell engulfment. Cell 147, 1442-1445.

Jais, A., Einwallner, E., Sharif, O., Gossens, K., Lu, T.T., Soyal, S.M., Medgyesi, D., Neureiter, D., Paier-Pourani, J., Dalgaard, K., et al. (2014). Heme oxygenase-1 drives metaflammation and insulin resistance in mouse and man. Cell 158, 25-40.

Jha, A.K., Huang, S.C., Sergushichev, A., Lampropoulou, V., Ivanova, Y., Loginicheva, E., Chmielewski, K., Stewart, K.M., Ashall, J., Everts, B., et al. (2015). Network integration of parallel metabolic and transcriptional data reveals metabolic modules that regulate macrophage polarization. Immunity 42, 419-430.

Johnson, M.O., Wolf, M.M., Madden, M.Z., Andrejeva, G., Sugiura, A., Contreras, D.C., Maseda, D., Liberti, M.V., Paz, K., Kishton, R.J., Johnson, M.E., de Cubas, A.A., Wu, P., Li, 
G., Zhang, Y., Newcomb, D.C., Wells, A.D., Restifo, N.P., Rathmell, W.K., Locasale, J.W., Davila, M.L., Blazar, B.R., Rathmell, J.C. Distinct Regulation of Th17 and Th1 Cell Differentiation by Glutaminase-Dependent Metabolism. Cell. 2018 Dec 13;175(7):17801795.e19.

Kim, K.W., Williams, J.W., Wang, Y.T., Ivanov, S., Gilfillan, S., Colonna, M., Virgin, H.W., Gautier, E.L., and Randolph, G.J. (2016). MHC II+ resident peritoneal and pleural macrophages rely on IRF4 for development from circulating monocytes. J Exp Med 213, 19511959.

Kojima, Y., Weissman, I.L., and Leeper, N.J. (2017). The Role of Efferocytosis in Atherosclerosis. Circulation 135, 476-489.

Kuhn, R., Schwenk, F., Aguet, M., and Rajewsky, K. (1995). Inducible gene targeting in mice. Science 269, 1427-1429.

Liu, P.S., Wang, H., Li, X., Chao, T., Teav, T., Christen, S., Di Conza, G., Cheng, W.C., Chou, C.H., Vavakova, M., et al. (2017). alpha-ketoglutarate orchestrates macrophage activation through metabolic and epigenetic reprogramming. Nat Immunol 18, 985-994.

Morioka, S., Perry, J.S.A., Raymond, M.H., Medina, C.B., Zhu, Y., Zhao, L., Serbulea, V., Onengut-Gumuscu, S., Leitinger, N., Kucenas, S., et al. (2018). Efferocytosis induces a novel SLC program to promote glucose uptake and lactate release. Nature 563, 714-718.

Nicklin, P., Bergman, P., Zhang, B., Triantafellow, E., Wang, H., Nyfeler, B., Yang, H., Hild, M., Kung, C., Wilson, C., et al. (2009). Bidirectional transport of amino acids regulates mTOR and autophagy. Cell 136, 521-534.

O'Neill, L.A., and Artyomov, M.N. Itaconate: the poster child of metabolic reprogramming in macrophage function. Nat Rev Immunol. 2019 May;19(5):273-281

O'Neill, L.A., and Pearce, E.J. (2016). Immunometabolism governs dendritic cell and macrophage function. J Exp Med 213, 15-23.

Papathanassiu, A.E., Ko, J.H., Imprialou, M., Bagnati, M., Srivastava, P.K., Vu, H.A., Cucchi, D., McAdoo, S.P., Ananieva, E.A., Mauro, C., Behmoaras, J. BCAT1 controls metabolic reprogramming in activated human macrophages and is associated with inflammatory diseases. Nat Commun. 2017 Jul 12;8:16040.

Stipanuk, M.H and Caudill, M.A. Biochemical, physiological and molecular aspects of human nutrition. 2013 International Book Number: 978-0-323-44181-0.

Tabas, I. (2010). Macrophage death and defective inflammation resolution in atherosclerosis. Nat Rev Immunol 10, 36-46.

Tavakoli, S., Downs, K., Short, J.D., Nguyen, H.N., Lai, Y., Jerabek, P.A., Goins, B., Toczek, J., Sadeghi, M.M., Asmis, R. Characterization of Macrophage Polarization States Using Combined Measurement of 2-Deoxyglucose and Glutamine Accumulation: Implications for Imaging of Atherosclerosis. Arterioscler Thromb Vasc Biol. 2017 Oct;37(10):1840-1848.

Vats, D., Mukundan, L., Odegaard, J.I., Zhang, L., Smith, K.L., Morel, C.R., Wagner, R.A., Greaves, D.R., Murray, P.J., and Chawla, A. (2006). Oxidative metabolism and PGC-1beta attenuate macrophage-mediated inflammation. Cell Metab 4, 13-24.

Viaud, M., Ivanov, S., Vujic, N., Duta-Mare, M., Aira, L.E., Barouillet, T., Garcia, E., Orange, F., Dugail, I., Hainault, I., Stehlik, C., Marchetti, S., Boyer, L., Guinamard, R., Foufelle, F., Bochem, A., Hovingh, K.G., Thorp, E.B., Gautier, E.L., Kratky, D., Dasilva-Jardine, P., YvanCharvet, L. Lysosomal Cholesterol Hydrolysis Couples Efferocytosis to Anti-Inflammatory Oxysterol Production. Circ Res. 2018 May 11;122(10):1369-1384.

Wang, Y., Subramanian, M., Yurdagul, A. Jr., Barbosa-Lorenzi, V.C., Cai, B., de Juan-Sanz, J., Ryan, T.A., Nomura, M., Maxfield, F.R., Tabas, I. Mitochondrial Fission Promotes the Continued Clearance of Apoptotic Cells by Macrophages. Cell. 2017 Oct 5;171(2):331345.e22.

Yurdagul, A Jr., Subramanian, M., Wang, X., Crown, S.B., Ilkayeva, O.R., Darville, L., Kolluru, G.K., Rymond, C.C., Gerlach, B.D., Zheng, Z., et al. (2020). Macrophage Metabolism of Apoptotic Cell-Derived Arginine Promotes Continual Efferocytosis and Resolution of Injury. Cell Metab 31, 518-533.e10. 
Yun, T.J., Lee, J.S., Shim, D., Choi, J.H., and Cheong, C. (2017). Isolation and Characterization of Aortic Dendritic Cells and Lymphocytes in Atherosclerosis. Methods Mol Biol 1559, 419-437.

Yvan-Charvet, L., Pagler, T.A., Seimon, T.A., Thorp, E., Welch, C.L., Witztum, J.L., Tabas, I., and Tall, A.R. (2010). ABCA1 and ABCG1 protect against oxidative stress-induced macrophage apoptosis during efferocytosis. Circ Res 106, 1861-1869.

Zhang, S., Weinberg, S., DeBerge, M., Gainullina, A., Schipma, M., Kinchen, J.M., Ben-Sahra, I., Gius, D.R., Yvan-Charvet, L., Chandel, N.S., et al. (2019). Efferocytosis Fuels Requirements of Fatty Acid Oxidation and the Electron Transport Chain to Polarize Macrophages for Tissue Repair. Cell Metab 29, 443-456 e445. 


\section{Graphical Abstract}

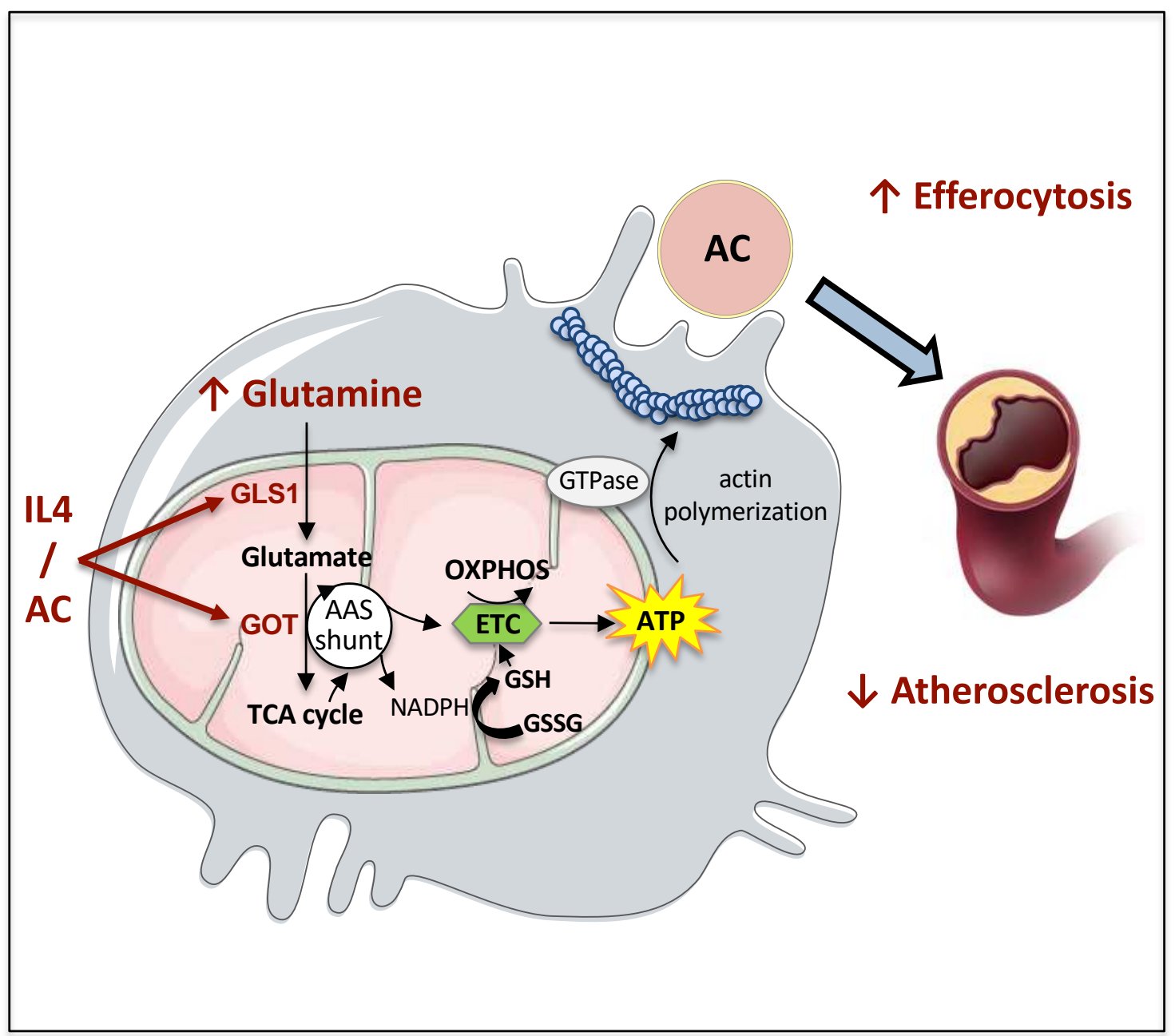


Figure 1

a
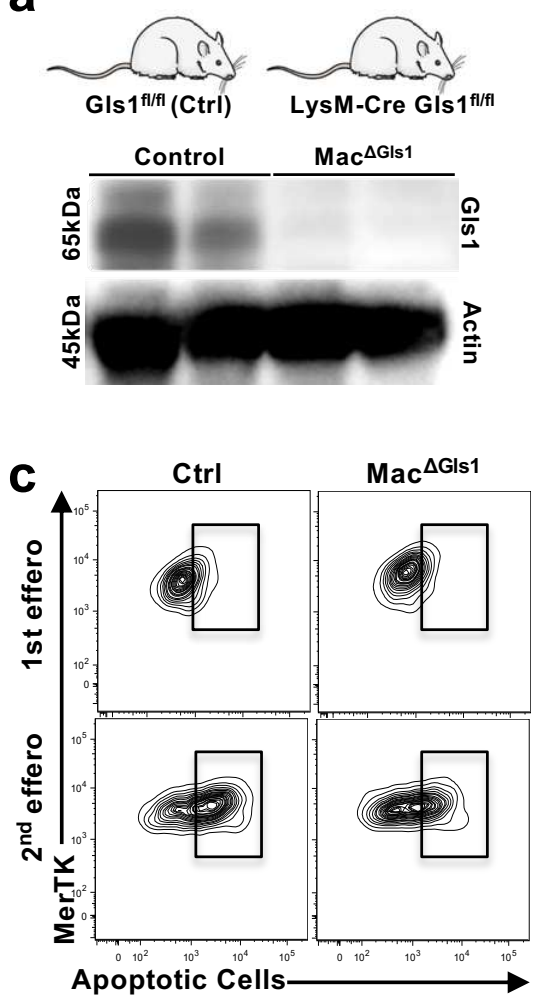
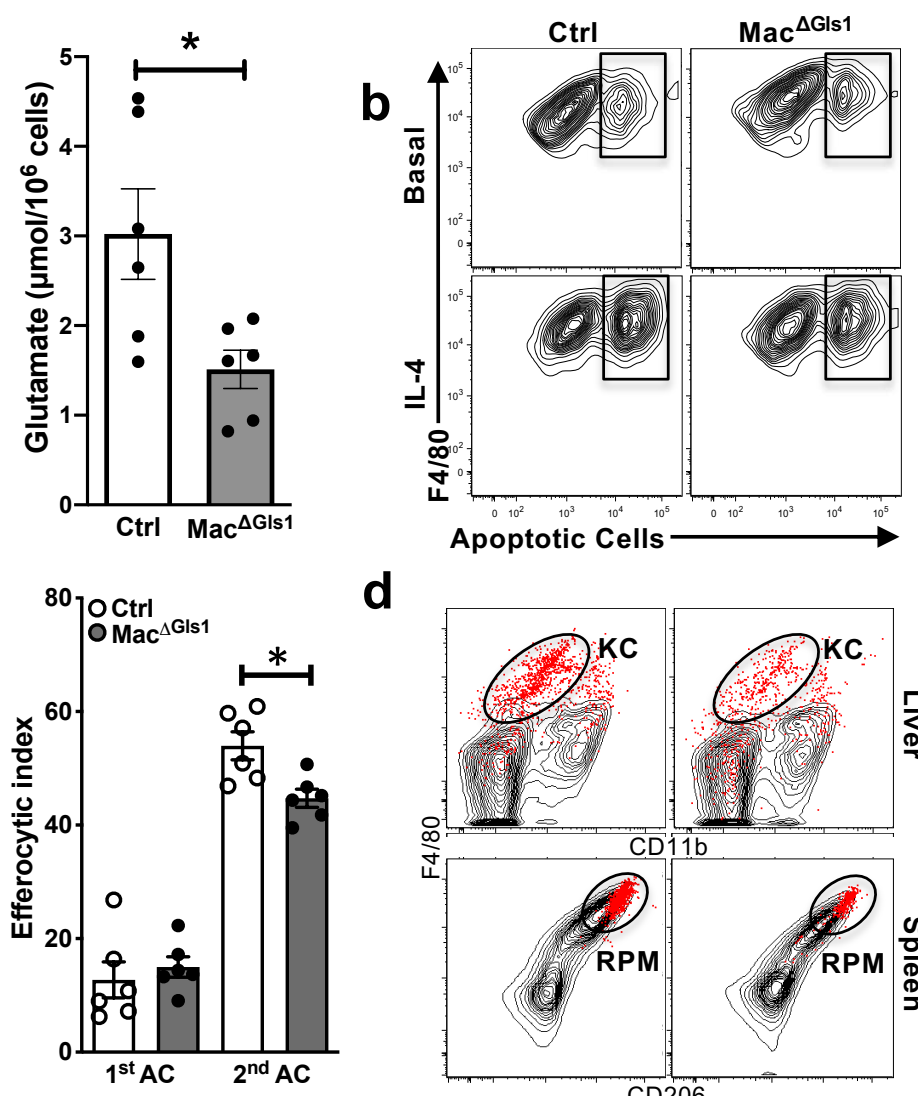

d

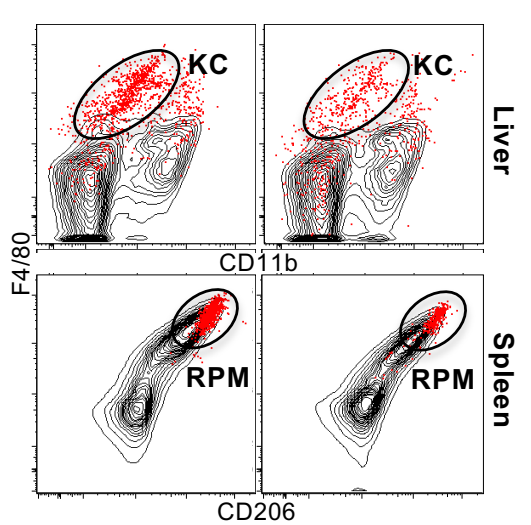

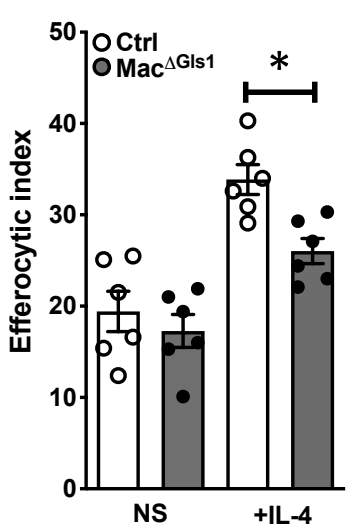

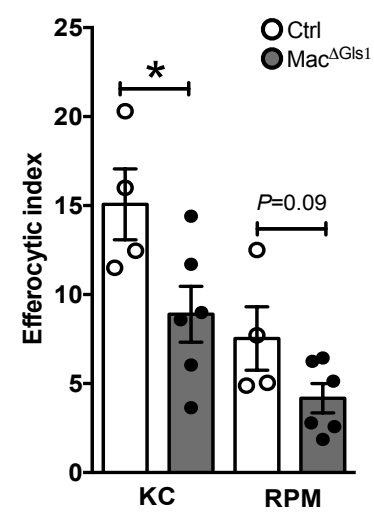




\section{Figure 2}
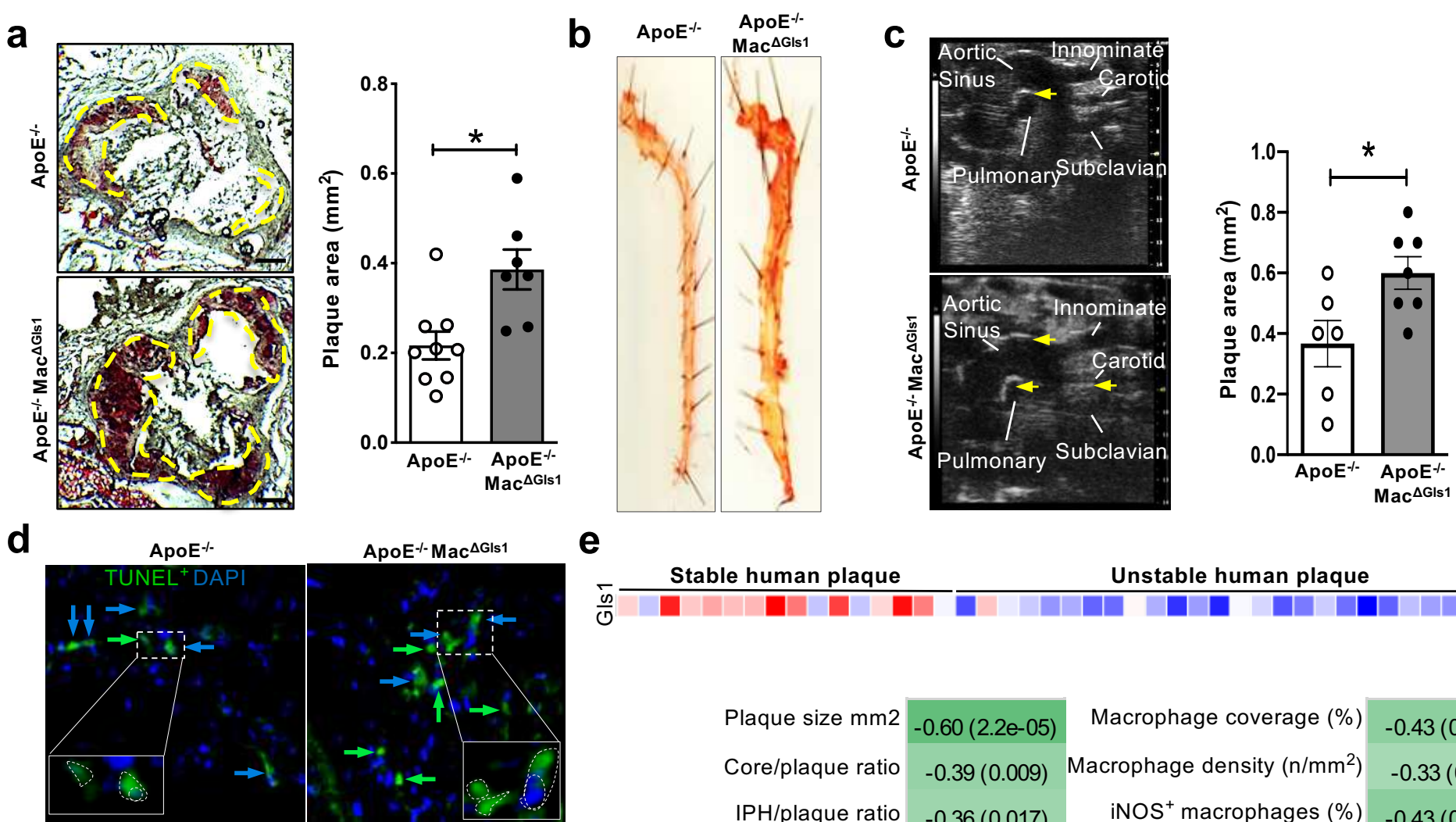

ApoE $^{-/-M a c}$ MGls1 $^{\Delta}$
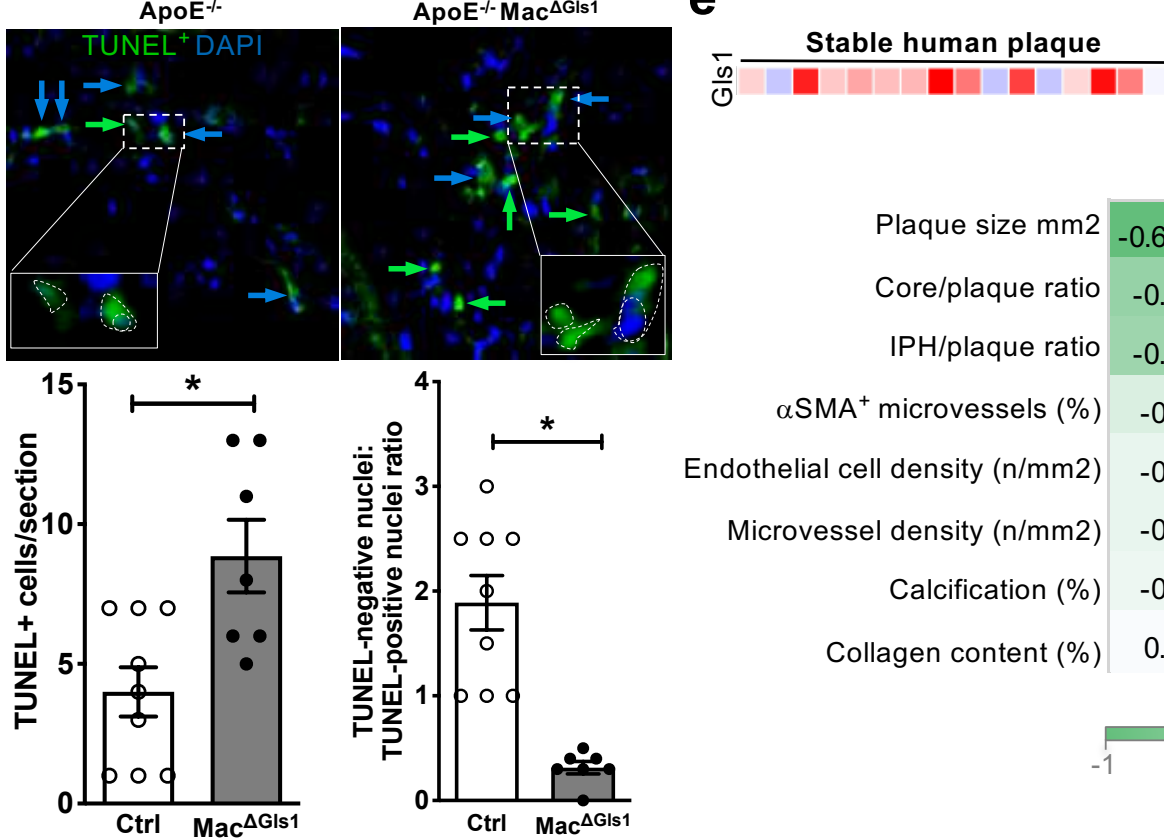

Unstable human plaque

\begin{tabular}{|c|c|c|c|}
\hline Plaque size $\mathrm{mm} 2$ & $-0.60(2.2 \mathrm{e}-05)$ & Macrophage coverage (\%) & $-0.43(0.004)$ \\
\hline Core/plaque ratio & $-0.39(0.009)$ & Macrophage density $\left(\mathrm{n} / \mathrm{mm}^{2}\right)$ & $-0.33(0.03)$ \\
\hline $\mathrm{IPH} /$ plaque ratio & $-0.36(0.017)$ & $\mathrm{iNOS}^{+}$macrophages (\%) & $-0.43(0.003)$ \\
\hline$\alpha \mathrm{SMA}^{+}$microvessels (\%) & $-0.12(0.44)$ & $\mathrm{MMP} 14^{+}$macrophages (\%) & $-0.24(0.11)$ \\
\hline Endothelial cell density $(\mathrm{n} / \mathrm{mm} 2)$ & $-0.06(0.70)$ & T cell density $(\mathrm{n} / \mathrm{mm} 2)$ & $-0.21(0.17)$ \\
\hline Microvessel density $(\mathrm{n} / \mathrm{mm} 2)$ & $-0.04(0.79)$ & $\mathrm{CD} 163^{+}$macrophages $(\%)$ & $-0.01(0.93)$ \\
\hline Calcification (\%) & $-0.04(0.79)$ & Giant Cells/CD68 & $0.23(0.13)$ \\
\hline \multirow[t]{2}{*}{ Collagen content $(\%)$} & $0.01(0.96)$ & Arg $1^{+}$macrophages (\%) & $0.66(1.3 e-06)$ \\
\hline & Gls1 & & Gls1 \\
\hline
\end{tabular}


Figure 3
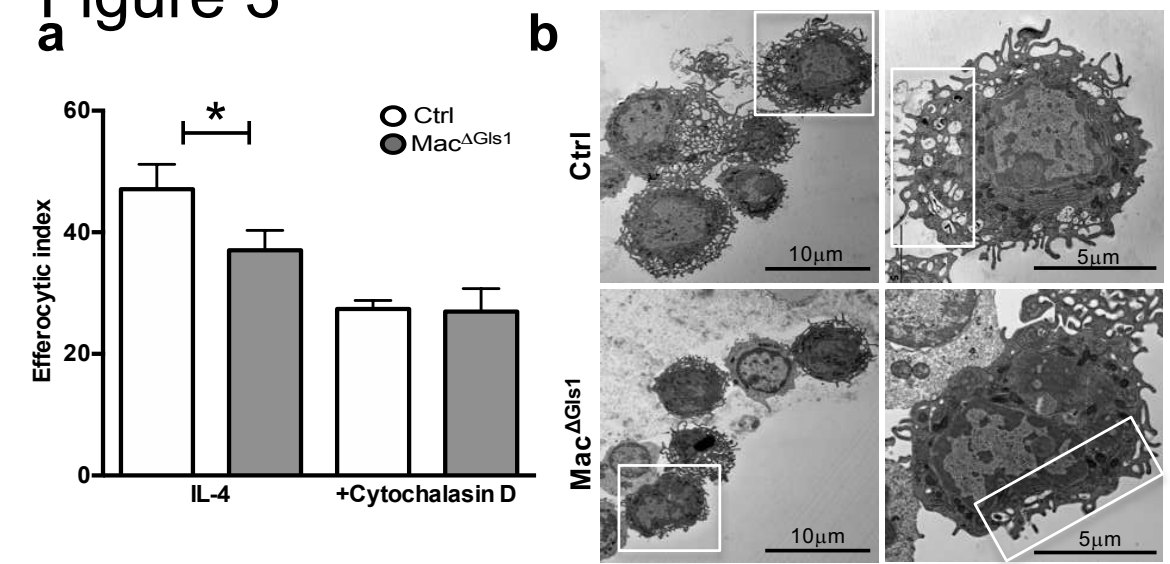

d

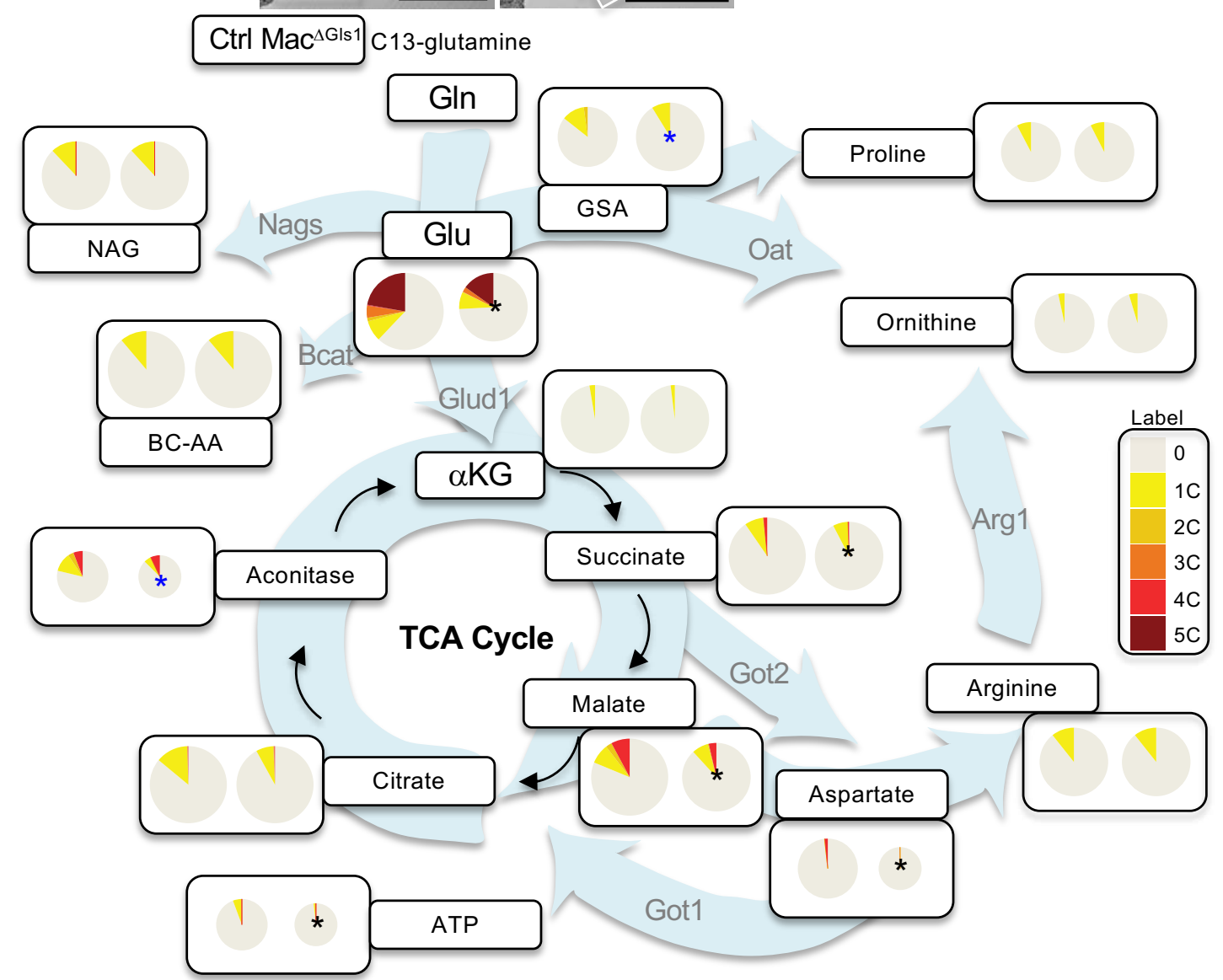

$\mathbf{e}_{50}$

O AC alone



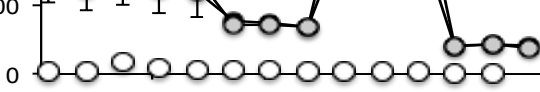

h

- Ctrl +AC
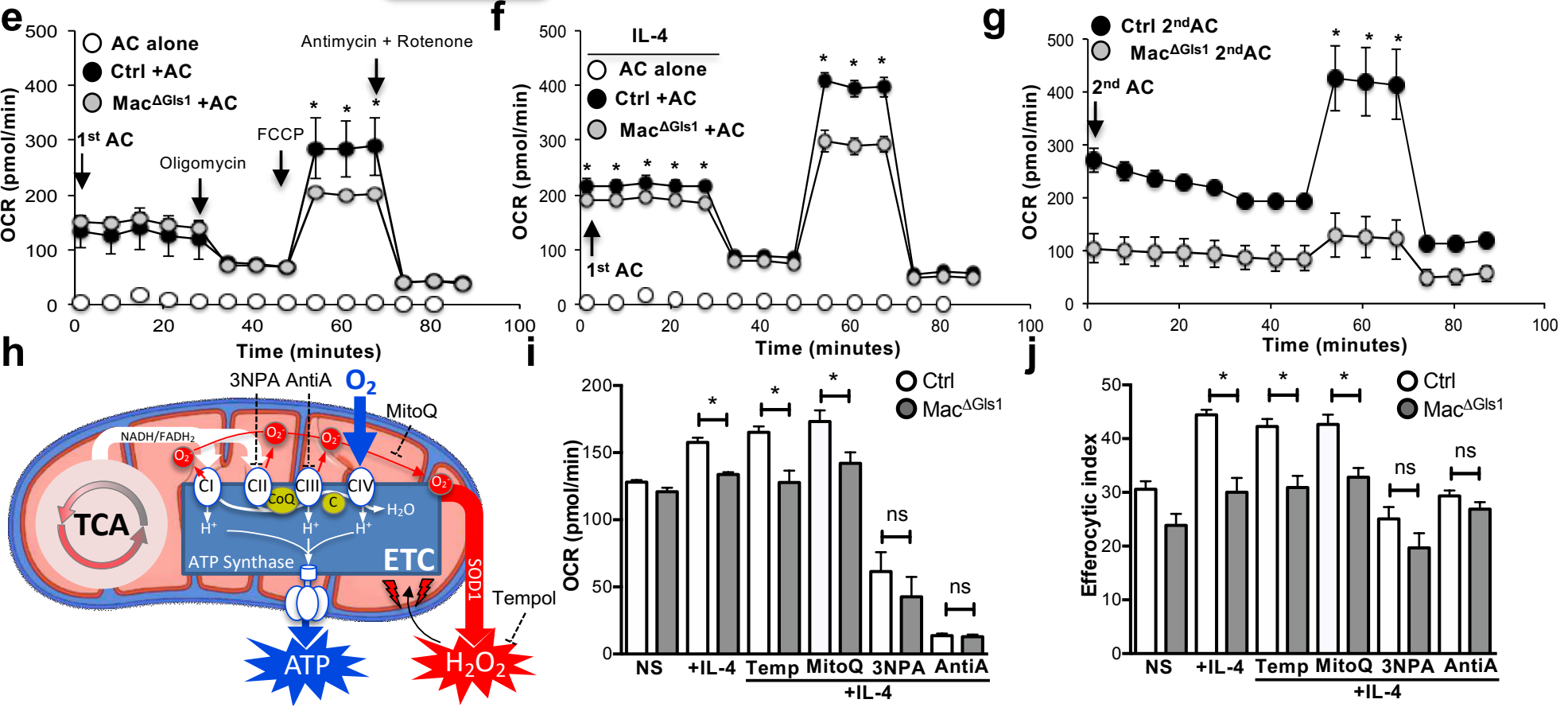
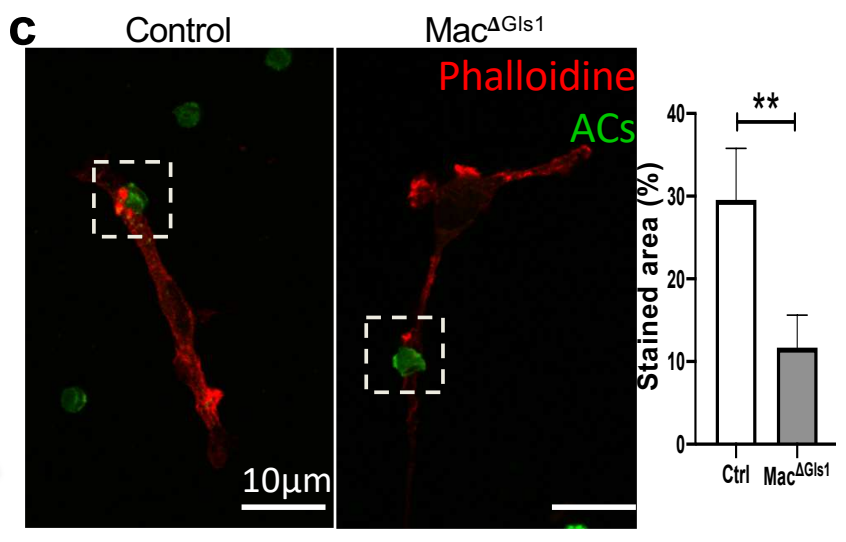


\section{Figure 4}

a

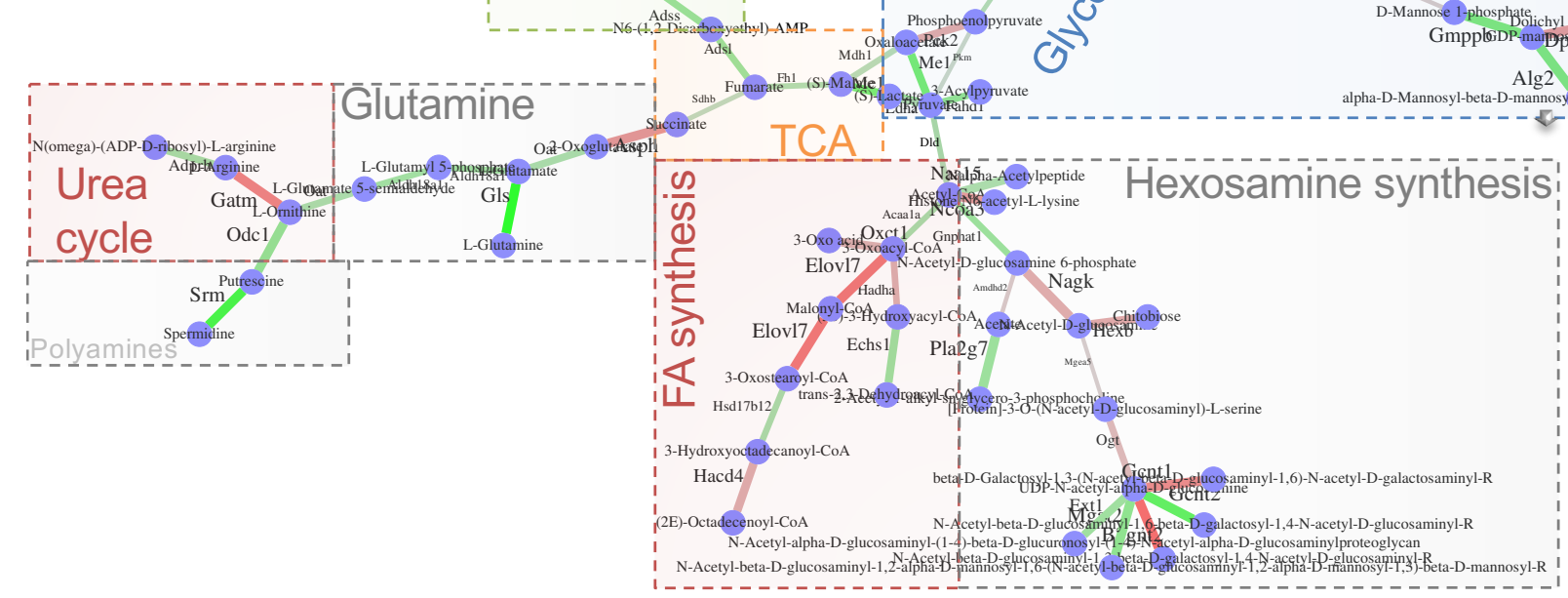

b c $\quad$ c $\quad$ d
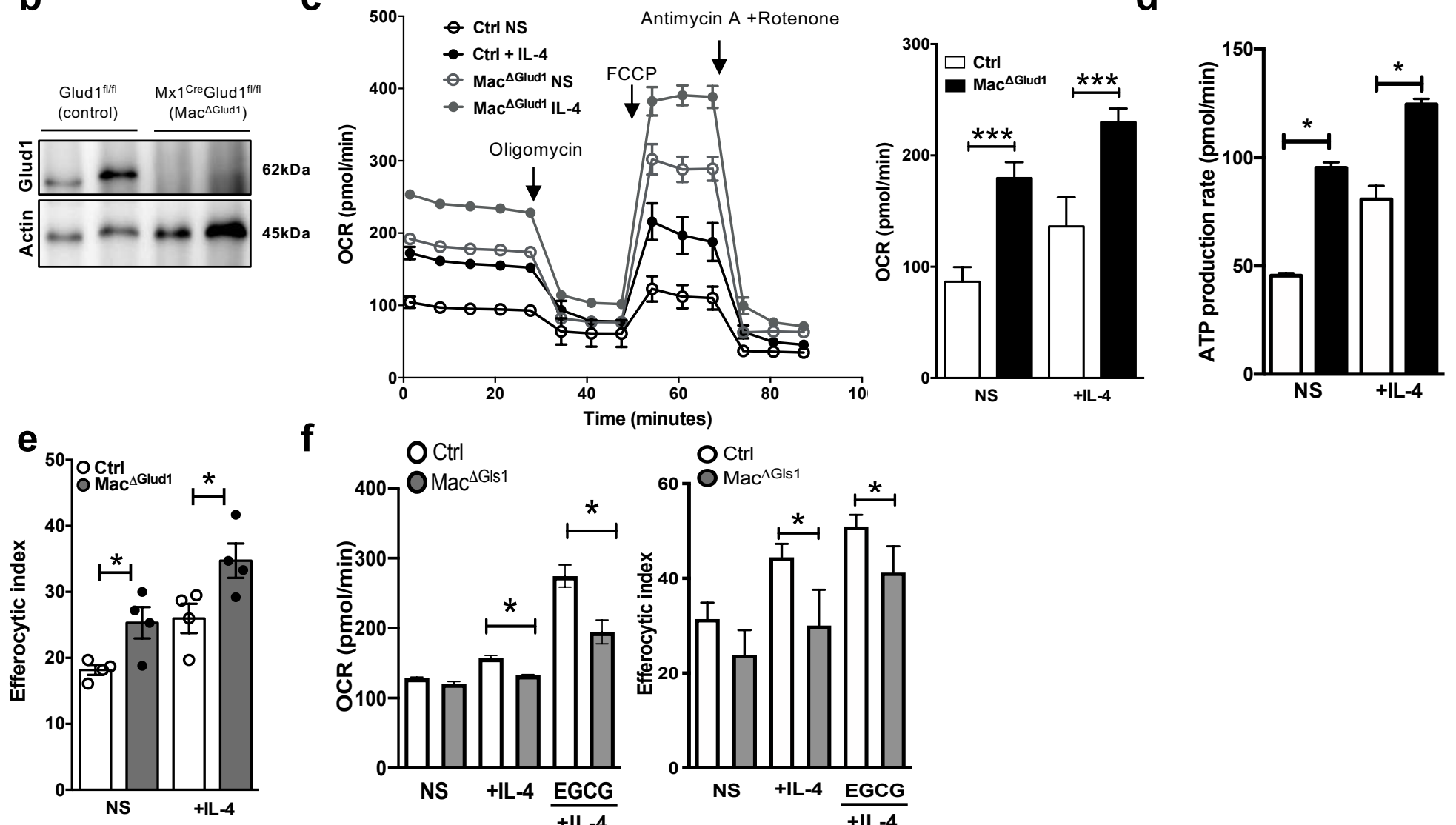

f
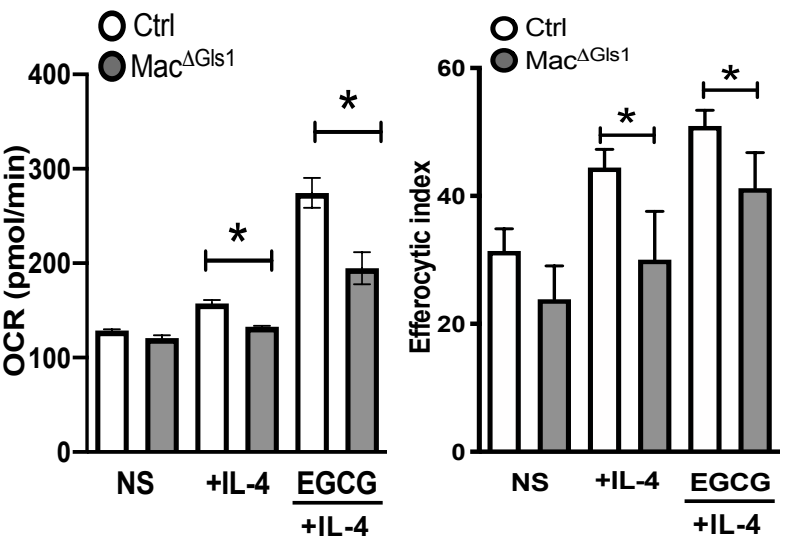
Figure 5

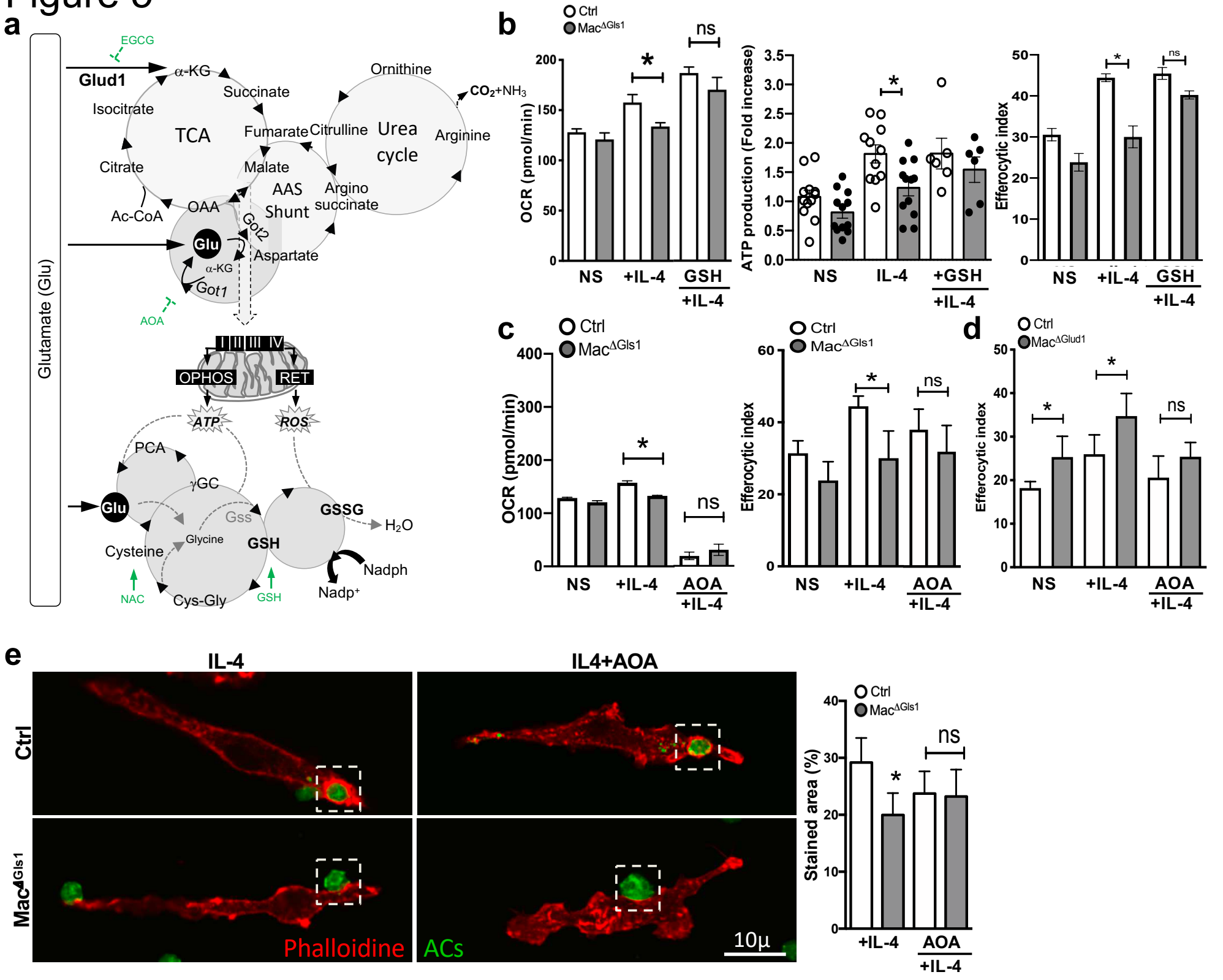


a

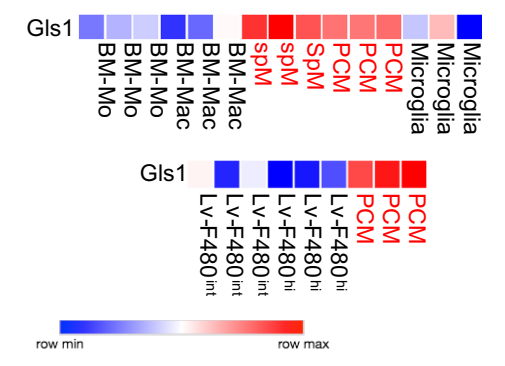

b

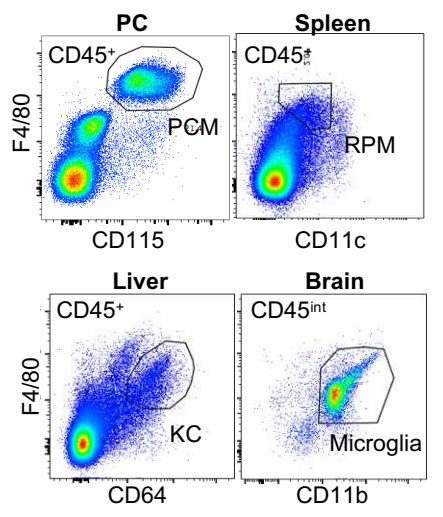

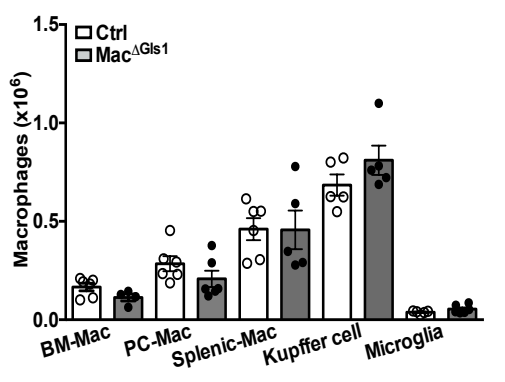

d
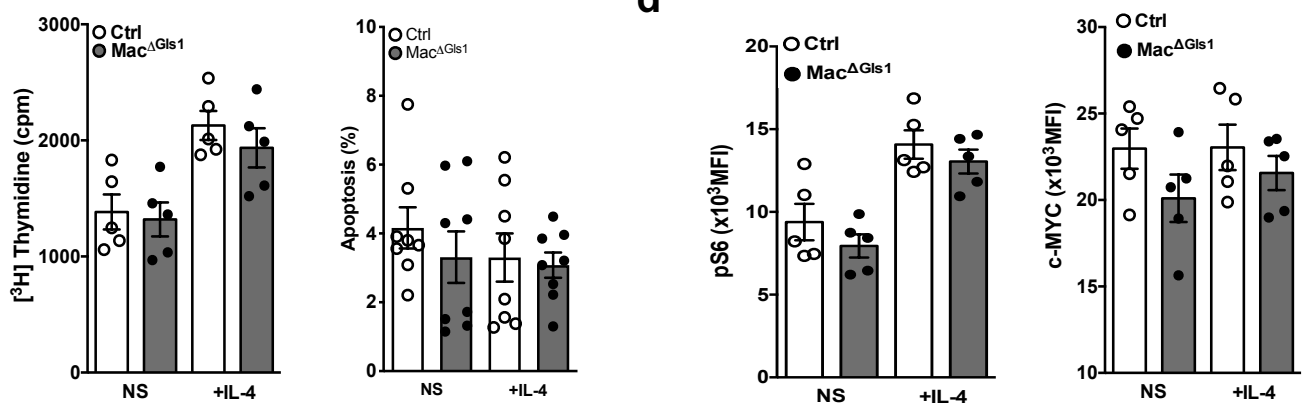

e

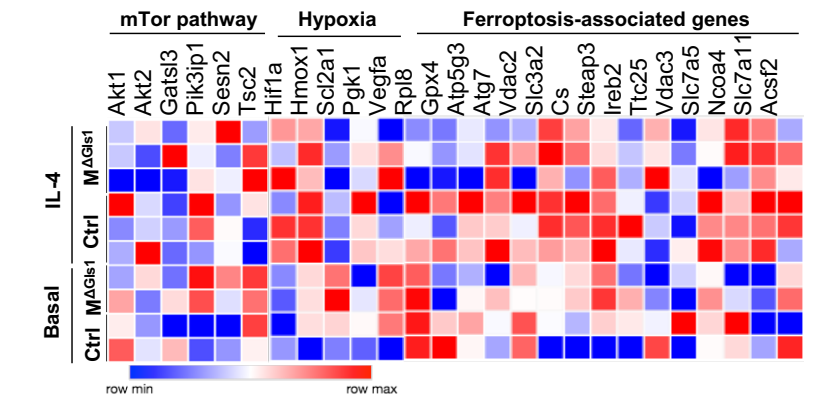

f

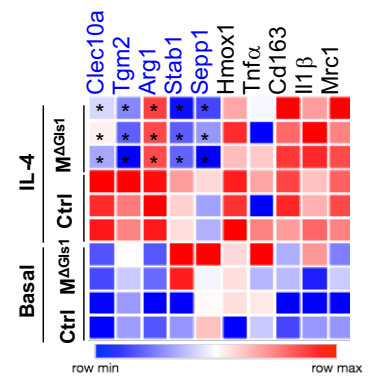

g
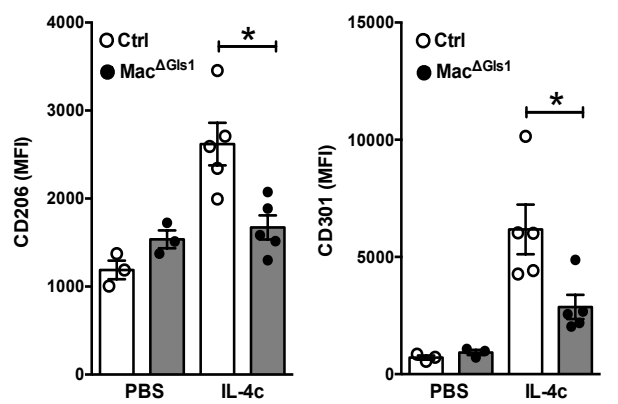

h
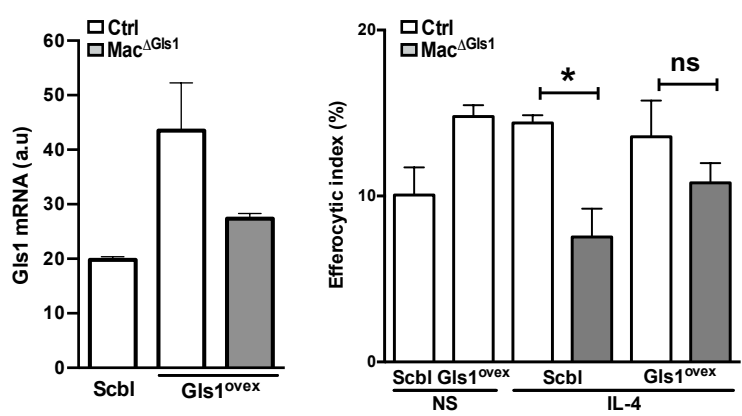

Figure S1: GLS1 is dispensable for macrophage homeostasis but supports macrophage effector and clearance functions. (a) Comparative analysis of Gls1 mRNA expression in different macrophage populations from a publicly available dataset (Immgen). (b) Macrophage population gating strategy (left) and numbers (right) measured by flow cytometry in multiple mouse tissues (bone marrow, peritoneal cavity, spleen, liver and brain). $n=5-6$ (c) $\left[{ }^{3} \mathrm{H}\right]-$ Thymidine incorporation (left) and apoptosis percentage (right) in control or Mac ${ }^{\Delta G \mid s 1}$ BMDMs at steady state or after an overnight stimulation with IL-4. (d) Phospho-S6 (left) and c-myc (right) expression measured by flow cytometry in control or Mac ${ }^{\Delta G l s 1}$ BMDMs with or without overnight IL-4 stimulation. (e) RNAseq of control or Mac ${ }^{\Delta G l s 1}$ cell sorted PCMs at steady state or after IL-4 stimulation. (f) RNAseq analysis with focus on alternatively activated genes in control or Mac ${ }^{\Delta \mathrm{Gls} 1}$ PCMs stimulated overnight or not with IL-4. (g) CD206 and CD301 
expression by flow cytometry in mice injected i.p. with PBS or IL-4-complex. (h) qPCR quantification (left) and efferocytic index (right) of Gls1 lentivirus overexpression in control or $\mathrm{Mac}^{\Delta \mathrm{Gls} 1}$ BMDMs stimulated overnight with IL-4. All values are mean \pm SEM and are representative of at least one experiment ( $n=3-13$ independent animals). ${ }^{*} P<0.05$ 


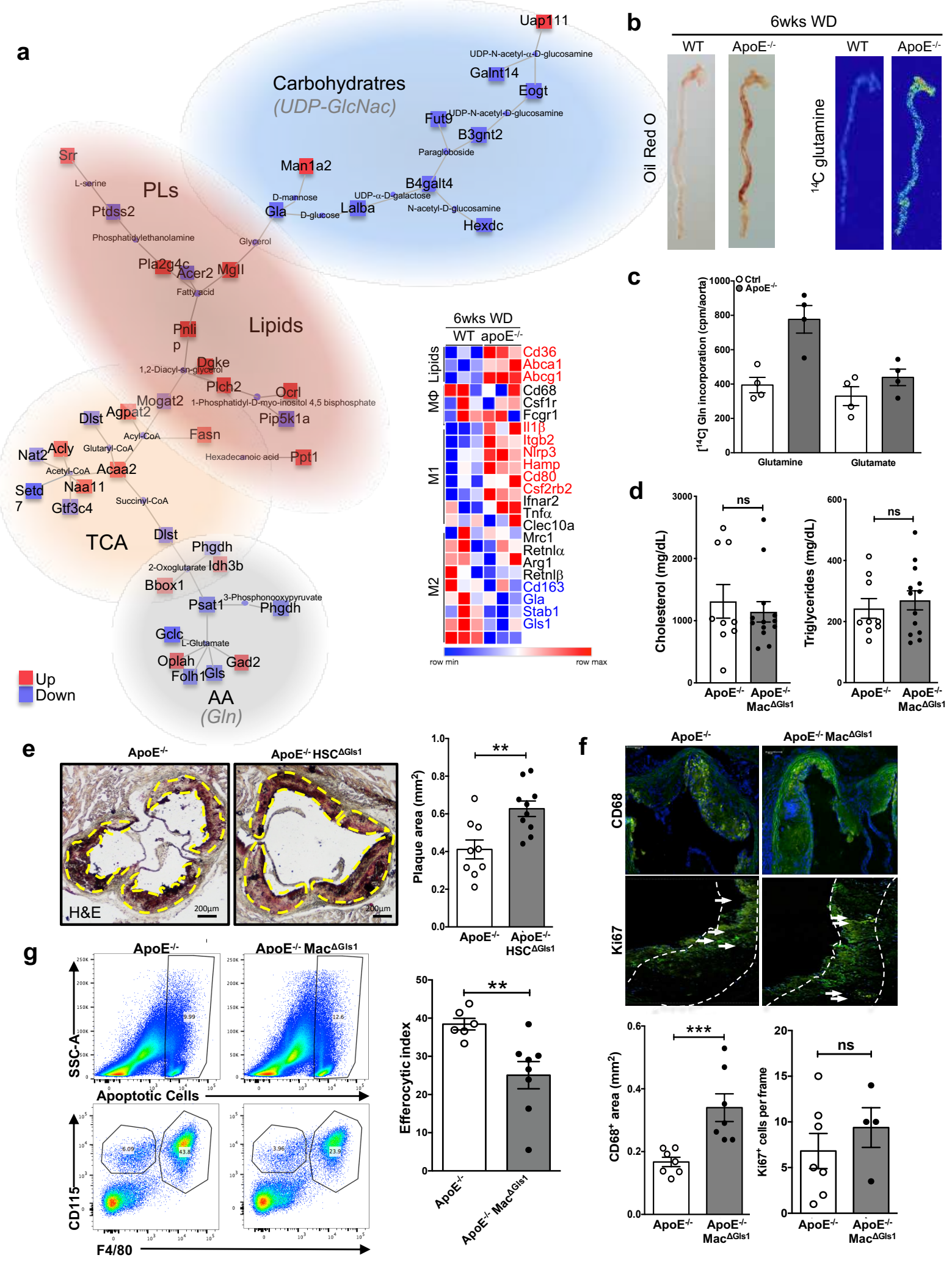

Figure S2: Atherosclerosis development relies on GLS1-dependent glutaminolyis. (a) Metabolic pathway (left) and RNAseq analysis (right) of $\mathrm{ApoE}^{-/-}$versus WT mouse aortas (6 weeks on WD) performed with Phantasus software (GSE10000). (b) Oil red O staining (left) or ${ }^{14} \mathrm{C}$ glutamine accumulation after i.v. injection (right) in descending aortas extracted from WT and $\mathrm{ApoE}^{-/-}$mice maintained on a WD for 6 weeks. (c) ${ }^{14} \mathrm{C}$ glutamine incorporation in aortas obtained from $\mathrm{ApoE}^{-/-}$and $\mathrm{ApoE}^{-/-} \mathrm{Mac}^{\Delta \mathrm{Gls} 1}$ mice fed for 6 weeks on WD. (d) Cholesterol (left) and triglyceride (right) content in plasma of $\mathrm{ApoE}^{-/-}$and $\mathrm{ApoE}^{-/-} \mathrm{Mac}^{\Delta \mathrm{Gls} 1}$ mice. (e) 
Representative sections (left) and quantification (right) of aortic plaques from $\mathrm{ApoE}^{-/-}$or $\mathrm{ApoE}^{-}$ ${ }^{1-} \mathrm{HSC}^{\Delta \mathrm{Gls} 1}$ mice (12 weeks WD) stained for Oil Red O and Hematoxylin Eosin. Scale bar: $200 \mu \mathrm{m}$. (f) Representative sections (top) and quantification (bottom) of aortic plaques from $\mathrm{ApoE}^{-/-}$or ApoE ${ }^{-/-} \mathrm{Mac}^{\Delta \mathrm{Gls} 1}$ mice (12 weeks WD) stained for CD68 and Ki67. (g) Gating strategy (left) and quantification (right) of $\mathrm{PCM}$ efferocytic index in $\mathrm{ApoE}^{-/-}$and $\mathrm{ApoE}^{-/-} \mathrm{Mac}^{\Delta \mathrm{Gls}^{\prime}}$ mice 1hour after labeled ACs i.p. injection. All values are mean \pm SEM and are representative of at least one experiment $(\mathrm{n}=4-13)$. ${ }^{* *} P<0.005 ;{ }^{* * *} P<0.0005$ compared to control. 


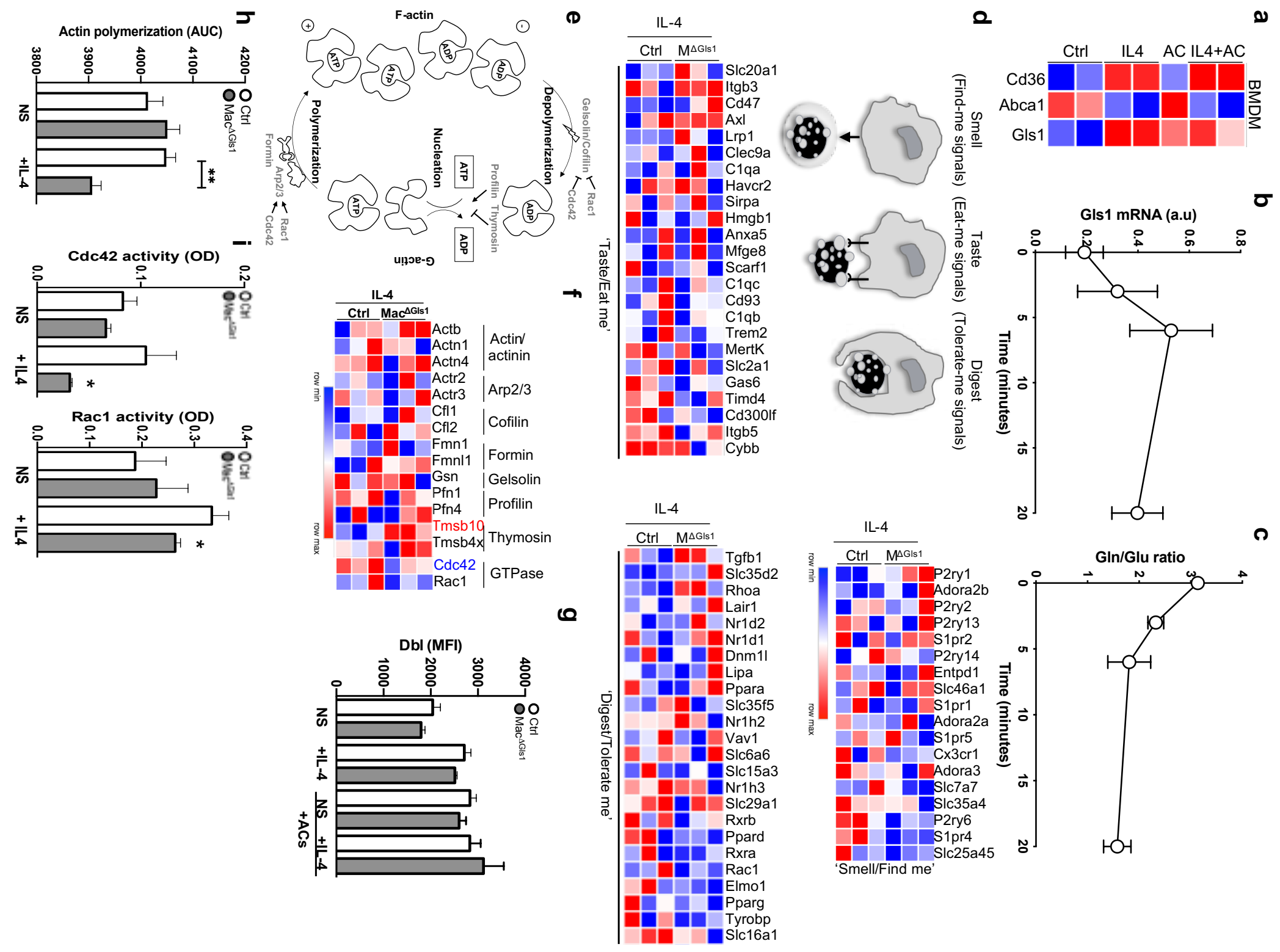



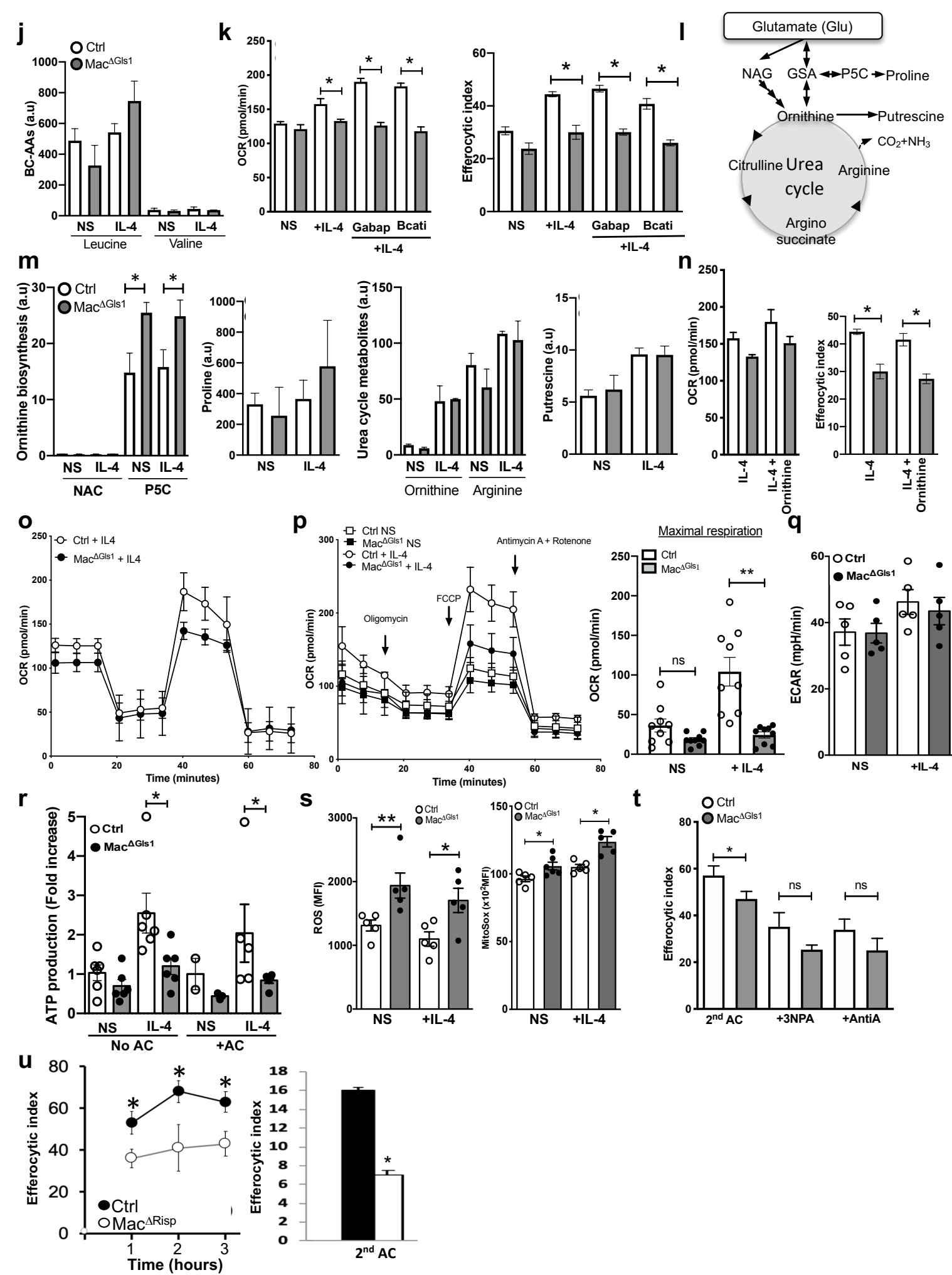

Figure S3: Gls1-deficient macrophages have a defect in ATP-dependent corpse engulfment. (a) Publicly available gene expression datasets analysis of macrophages ingesting apoptotic cells (GSE98169). (b) Gls1 mRNA expression and (c) Glutamine/Glutamate ratio during efferocytosis in control BMDMs in a time course experiment. (d) Schematic representation of efferocytosis steps and RNAseq analysis of "find-me", "eatme" and "tolerate-me" signals in control and Mac ${ }^{\Delta \mathrm{Gls} 1}$ PCMs stimulated overnight with IL-4. (e) Schematic representation of actin polymerization and depolarization. (f) RNAseq analysis with 
focus on F-actin dynamic regulators in control or $\mathrm{Mac}^{\Delta \mathrm{Gls} 1} \mathrm{PCMs}$ stimulated overnight with IL4. (g) Dbl expression quantified by flow cytometry in control or IL-4-treated macrophages that were incubated $\pm A C s$ for $45 \mathrm{~min}$. (h) Actin polymerization assay in control or Mac ${ }^{\Delta \mathrm{Gls} 1}$ BMDMs in basal condition or following overnight IL-4 stimulation. (i) Cdc42 and Rac1 activity assays in control or Mac ${ }^{\Delta \mathrm{Gls} 1} \mathrm{BMDMs}$ in basal condition or stimulated overnight with IL-4. (j) Leucine and Valine levels by liquid chromatography-mass spectrometry in control or Mac ${ }^{\triangle G \mid s 1}$ BMDMs at steady state or after-overnight IL-4 stimulation. (k) OCR (left panel) and efferocytic index (right panel) in control or Mac ${ }^{\Delta \mathrm{Gls} 1} \mathrm{BMDMs}$ in basal conditions or stimulated overnight with IL-4 and +/- Gabapentin or Bcat inhibitor. (I) Schematic representation of glutamate utilization in the urea cycle. (m) Quantification of NAG, P5C, proline, ornithine, arginine and putrescine levels by liquid chromatography-mass spectrometry in control or Mac ${ }^{\Delta \mathrm{Gls} 1} \mathrm{BMDMs}$ in basal condition or following overnight IL-4 treatment. (n) OCR quantification (left) and efferocytic index (right) of control or Mac ${ }^{\Delta G \mathrm{Is} 1}$ BMDMs treated overnight with IL-4 and ornithine. (o) OCR measured by Seahorse in control and Mac ${ }^{\Delta G l s 1}$ PCMs. ( $p$ ) OCR (left panel) and maximal respiration quantification (right panel) measured by Seahorse on control or $\mathrm{Mac}^{\Delta \mathrm{Gls} 1} \mathrm{BMDMs}$ with or without IL-4 stimulation. (q) ECAR measured by Seahorse in control or Mac ${ }^{\Delta G l s 1}$ BMDMs with or without overnight IL-4 stimulation. (r) ATP production measured by Seahorse in control or Mac $^{\Delta G l s 1}$ BMDMs after one or no round of efferocytosis and +/- overnight IL-4 stimulation. (s) ROS (left) and Mitosox (right) quantification using flow cytometry in control or Mac ${ }^{\Delta \mathrm{Gls} 1} \mathrm{BMDMs}$ at steady state or following overnight IL-4 stimulation. (t) Efferocytic index in control or Mac ${ }^{\Delta \mathrm{Gls} 1}$ BMDMs after either 2 rounds of efferocytosis or 3NPA +IL-4 or Antimycin A + IL-4 treatments overnight. (u) Efferocytic index after one round efferocytosis quantified by microscopy (left) or after two round efferocytosis measured by flow cytometry (right) in control or Risp ${ }^{-/}$ macrophages. All values are mean \pm SEM and are representative of at least one experiment $(\mathrm{n}=3-9) .{ }^{*} P<0.05,{ }^{* *} P<0.005$ compared to control. 
a
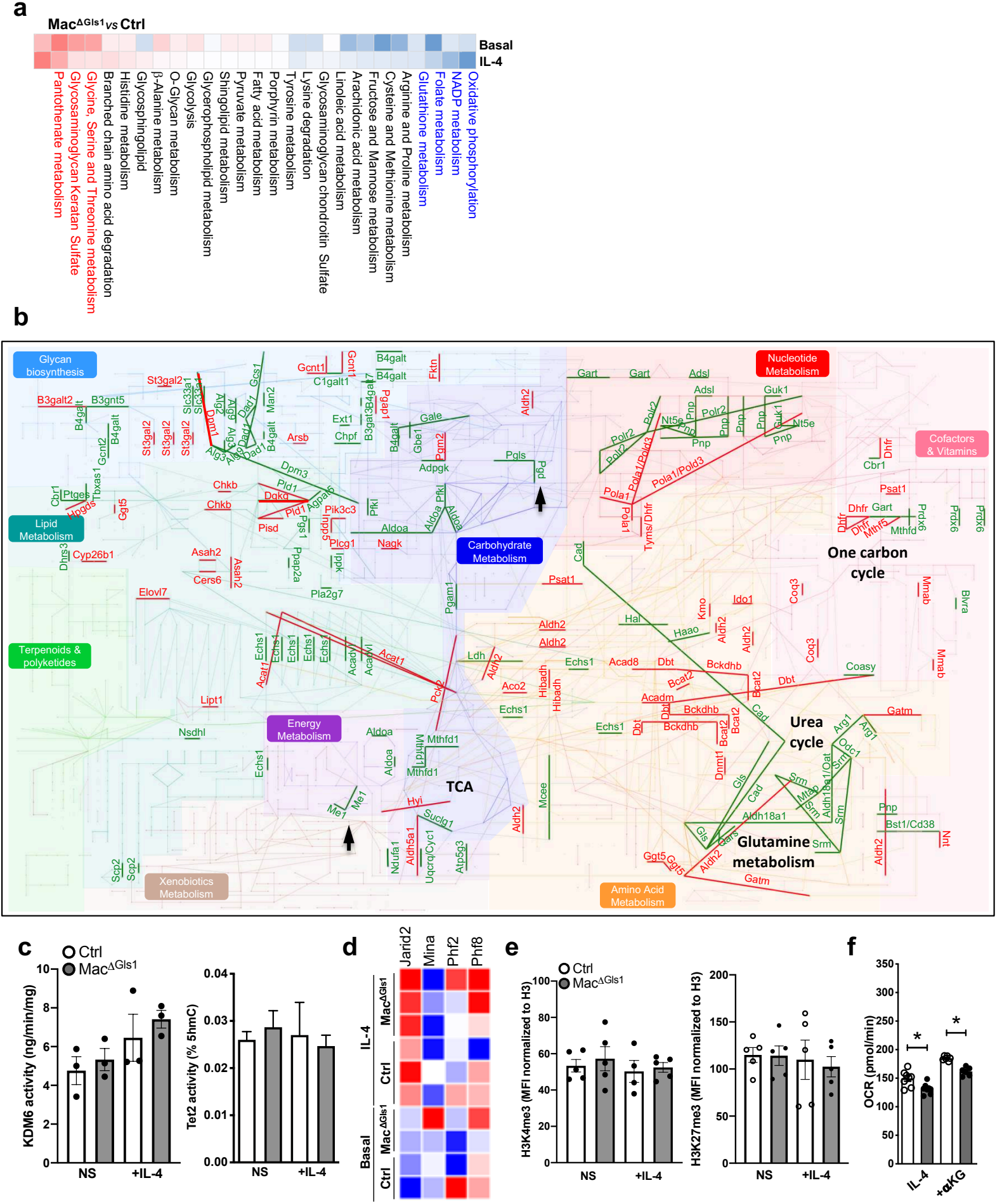

Figure S4: GLS1 is essential for macrophage mitochondrial oxidative phosphorylation and redox balance but not epigenetic reprogramming. (a) Pathway enrichment analysis of RNA-seq profiling in control or Mac ${ }^{\Delta \mathrm{Gls} 1} \mathrm{PCMs}$ in basal conditions or stimulated overnight with IL-4. (b) KEGG mapping with DreamBio from RNAseq data of alternatively activated Gls1deficient or sufficient PCMs. (c) KDM6 activity assay (left) and Tet2 activity assay (right) in control or Mac ${ }^{\Delta G l s 1}$ BMDMs in basal conditions or stimulated overnight with IL-4. (d) RNAseq analysis with focus on KDM6 target genes in control or Mac ${ }^{\Delta \mathrm{Gls} 1} \mathrm{PCMs}$ at steady state or after an overnight stimulation with IL-4. (e) Global histone trimethylation modification assessed by flow cytometry using $\mathrm{H} 3 \mathrm{~K} 4 \mathrm{me} 3$ and $\mathrm{H} 3 \mathrm{~K} 27 \mathrm{me} 3$ staining in control and $\mathrm{Mac}^{\Delta \mathrm{Gls} 1}$ BMDMs at 
steady state or stimulated overnight with IL-4. (f) OCR quantification in control or Mac ${ }^{\Delta \mathrm{Gls} 1}$ BMDMs stimulated overnight with IL-4 in presence or absence of aKetoglutarate. All values are mean \pm SEM and are representative of at least one experiment $(n=3-8) .{ }^{*} P<0.05$ compared to control. 


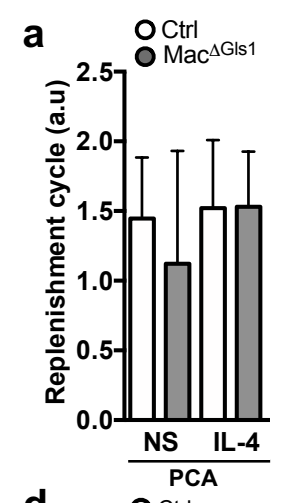

d

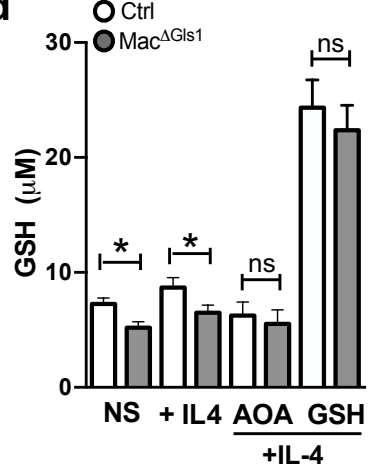

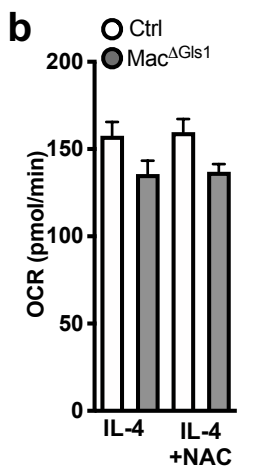

e

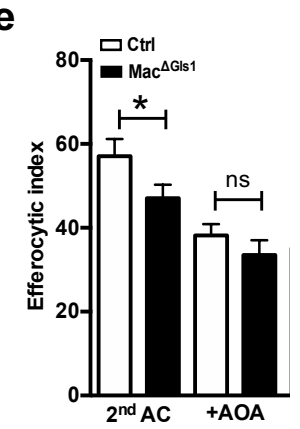

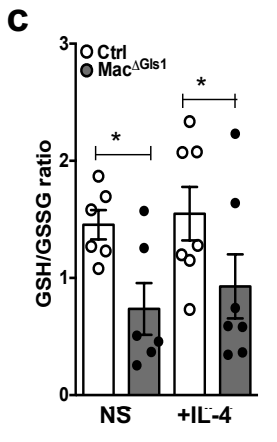
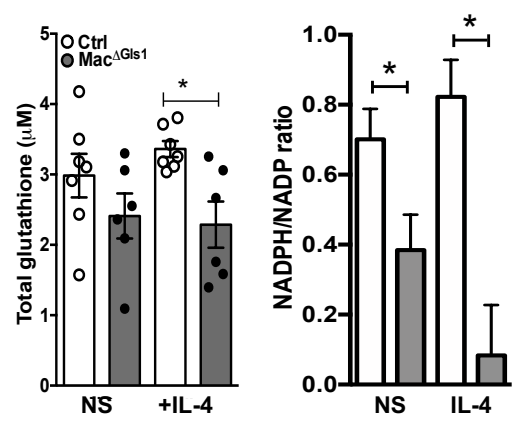

f

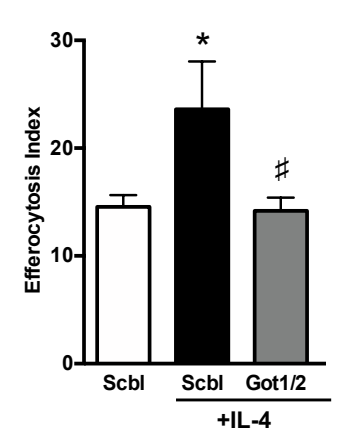

Figure S5: Non-canonical transaminase pathway is essential for actin dynamics and efferocytosis. (a) Effect of Gls1 deficiency on PCA (pyroglutamate), a glutathione precursor, as a read out of cellular GSH replenishment. (b) OCR quantification (left) and efferocytic index (right) in control or Mac ${ }^{\Delta \mathrm{Gls} 1}$ BMDMs treated overnight with IL-4 and IL-4 + N-Acetylcysteine (NAC). (c) GSH/GSSG ratio (left) and total glutathione concentration (middle) in control or Mac $^{\Delta G l s 1}$ BMDMs at steady state and NADPH/NADP ratio (right) analyzed by liquid chromatography-mass spectrometry in control or Mac ${ }^{\Delta \mathrm{Gls} 1} \mathrm{BMDMs}$ at steady state or after overnight IL-4 stimulation. (d) GSH concentration in control or Mac ${ }^{\Delta G \mid s 1} B M D M s$ at steady state or treated overnight with IL-4 and either AOA or GSH. (e) Efferocytic index in control or Mac ${ }^{\Delta \mathrm{Gls} 1}$ BMDMs after either 2 rounds of efferocytosis or AOA + IL-4 overnight treatment. (f) Efferocytic index in control or Mac ${ }^{\Delta \mathrm{Gls} 1} \mathrm{BMDMs}$ stimulated overnight or not with IL-4 and transfected with a scramble, or Got1/Got2 siRNA. All values are mean \pm SEM and are representative of at least one experiment $(n=3-6)$. ${ }^{*} P<0.05$ compared to control. 
a
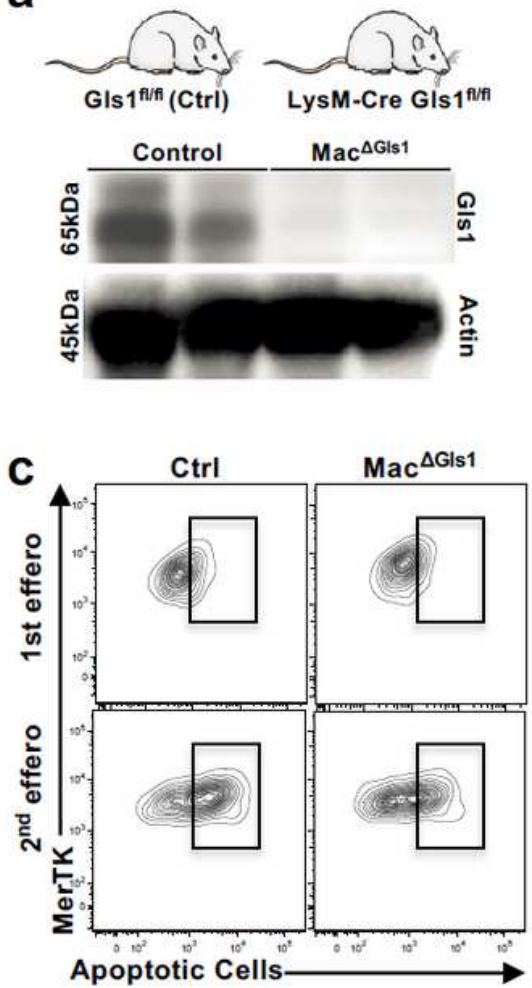
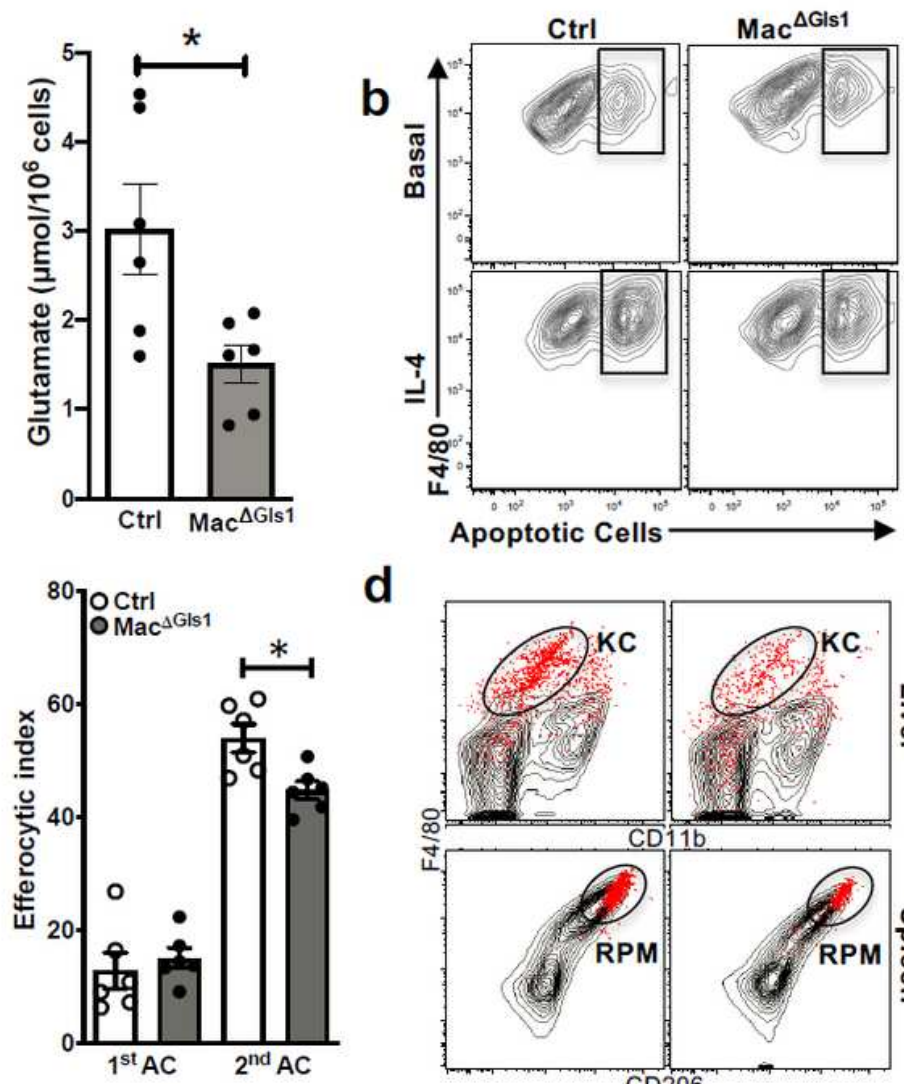

d

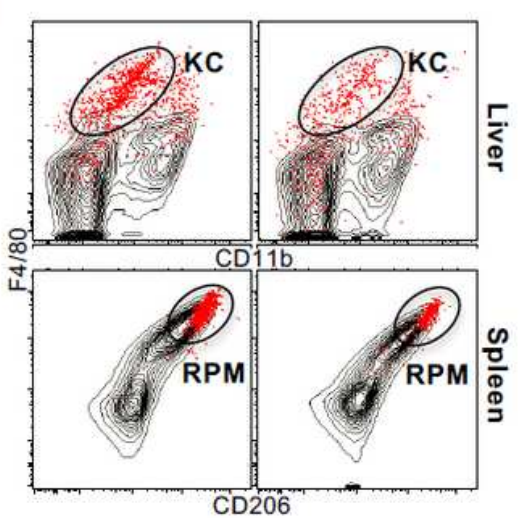

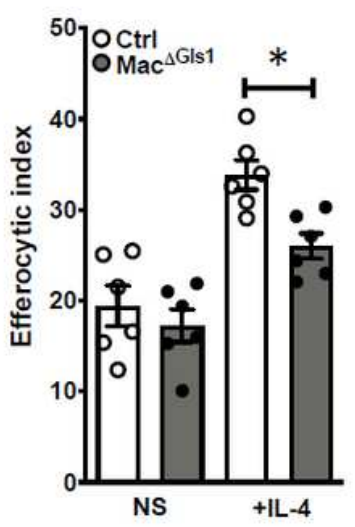

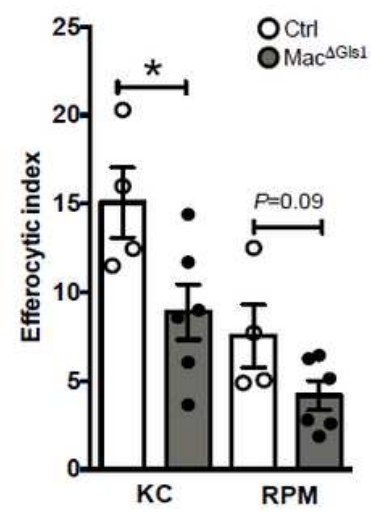

Figure 1

Macrophage-Gls1 deletion impairs efferocytosis in vitro and in vivo. (a) Western blot of Gls1 protein (left) and Glutamate levels (right) in Control or MacSGIs1 PCMs. (b) Efferocytic index gating strategy (left) and quantification (right) measured by flow cytometry after $45 \mathrm{~min}$ exposure with apoptotic cells (ACs) in BMDMs at steady state or after overnight IL-4 stimulation. Efferocytic index was calculated as follows: (number of macrophages with ACs/total number of macrophages) $\times 100$. (c) Efferocytic index gating strategy (Left) and quantification (right) measured by flow cytometry after one (45min) or two (45min + 1hour rest $+45 \mathrm{~min}$ ) incubations with ACs in control or Mac $\Delta$ Gls1 BMDMs. (d) Efferocytic index gating strategy (left) and quantification (right) measured by flow cytometry in control or Mac $\Delta$ Gls1 Kupffer cells (KC) and red pulp macrophages (RPM) after labelled apoptotic thymocytes i.v. injection. All values are mean \pm SEM and are representative of at least one experiment ( $n=4-6$ independent animals). ${ }^{*}<0.05$ compared to control. 


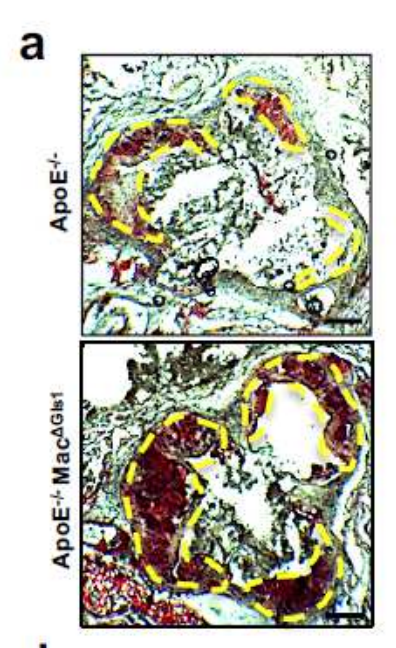

d
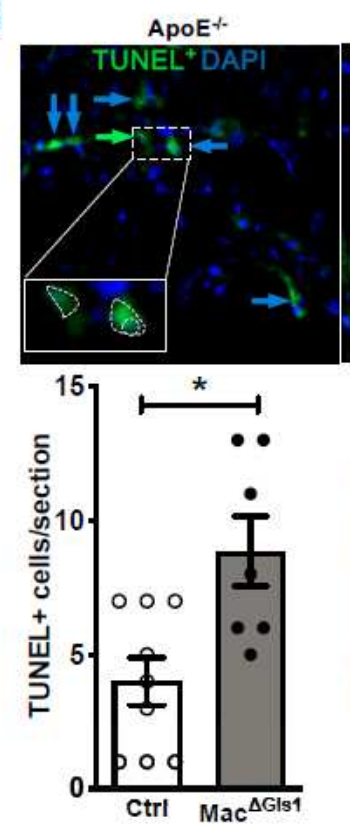

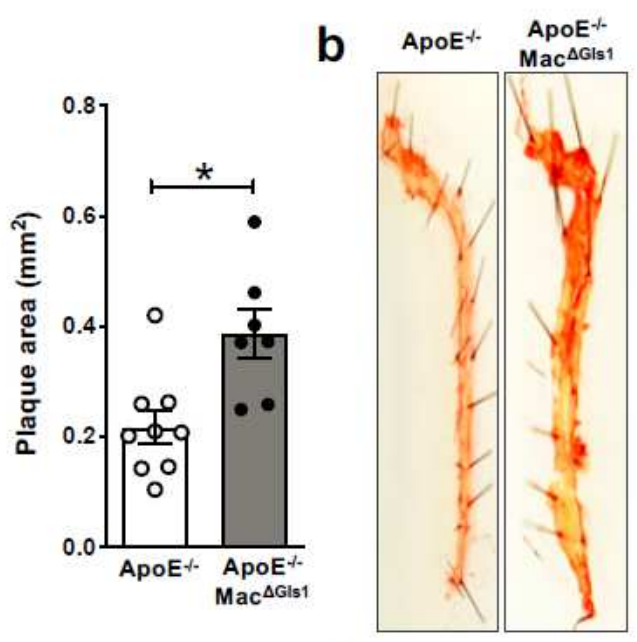

$A p o E^{\star-M a c}{ }^{\Delta G \mid s 1}$
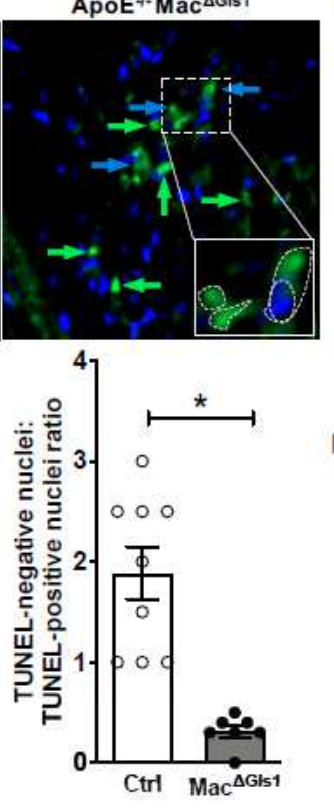

e
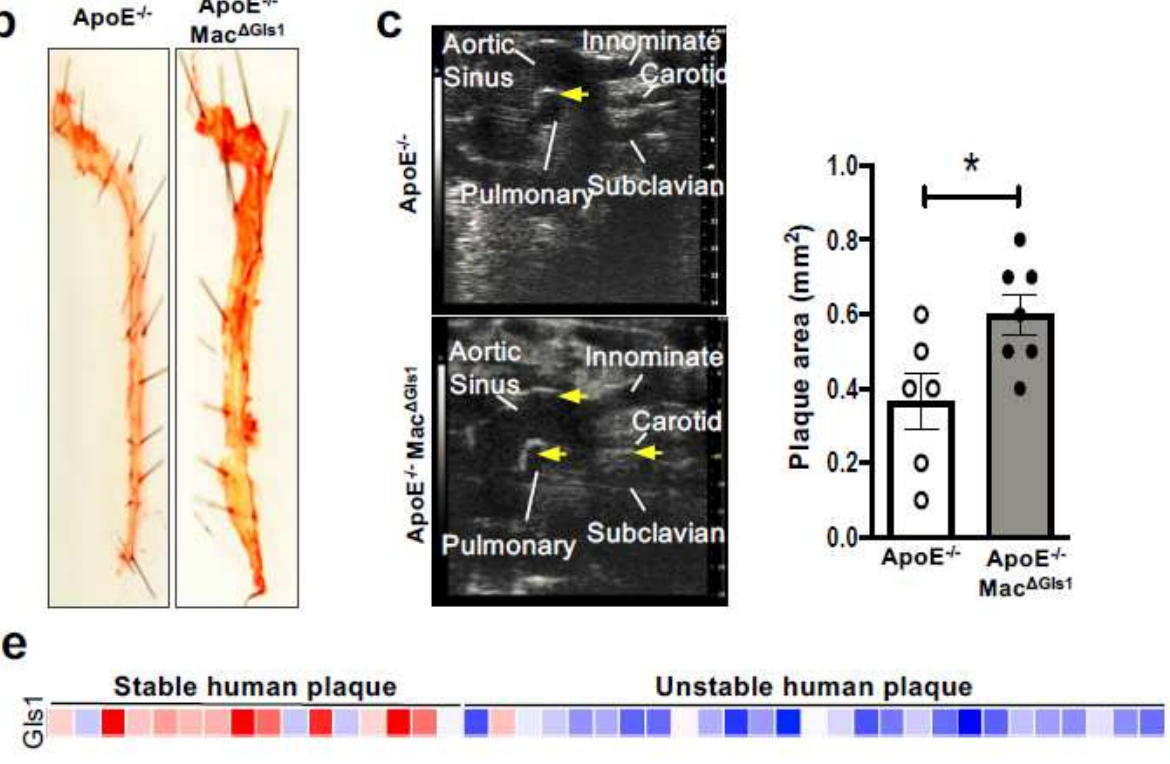

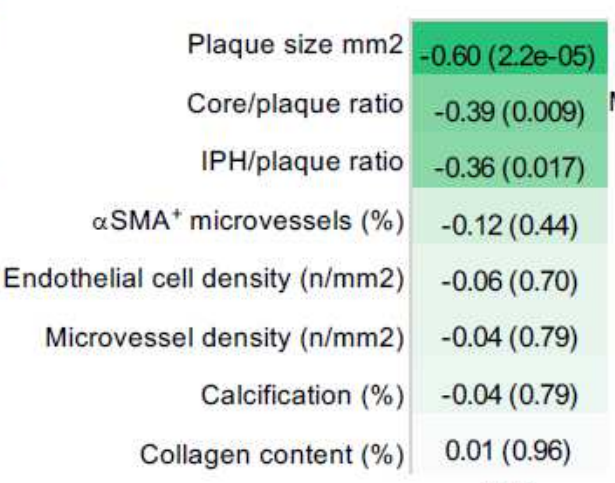

Gis1 \begin{tabular}{|l|l} 
Macrophage coverage (\%) & $-0.43(0.004)$
\end{tabular}

Macrophage density $\left(\mathrm{n} / \mathrm{mm}^{2}\right) \quad-0.33(0.03)$

iNOS $^{+}$macrophages (\%) $-0.43(0.003)$

MMP $14^{+}$macrophages (\%) $-0.24(0.11)$

T cell density $(\mathrm{n} / \mathrm{mm} 2) \quad-0.21(0.17)$

CD163 $3^{+}$macrophages $(\%) \quad-0.01(0.93)$

Giant Cells/CD68 $0.23(0.13)$

$\operatorname{Arg} 1^{+}$macrophages (\%) $0.66(1.3 \mathrm{e}-06)$

Gis1

\section{Figure 2}

Myeloid-Gls1 deletion impairs efferocytosis in the pathological process of atherosclerosis. (a) Representative sections (left) and quantification (right) of aortic plaques from ApoE-/- or ApoE-/-

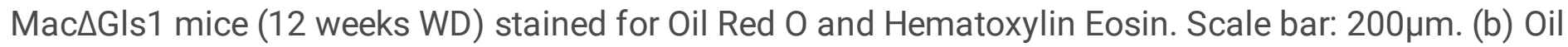
red 0 stained descending aortas from ApoE-/- or ApoE-/-Mac $\Delta$ Gls1 mice maintained on a WD for 12 weeks. (c) Echography (left) and quantification (right) of aortic plaques from ApoE-/- or ApoE-/Mac $\Delta$ GIs 1 mice fed for 12 weeks on WD. Arrows indicate plaque areas. (d) Representative images (top) and quantification (bottom) of TUNEL + cells in aortic plaques from ApoE-/- or ApoE-/- Mac $\Delta$ Gls1 mice (12 weeks WD). Green and blue arrow depict TUNEL negative nuclei and TUNEL-positive nuclei, respectively. (e) Correlation between Gls1 expression and human atherosclerotic plaque complexity in the Maastricht Pathology collection. All values are mean \pm SEM and are representative of at least one experiment $(n=6$ 9). ${ }^{*}<0.05$ compared to control. 

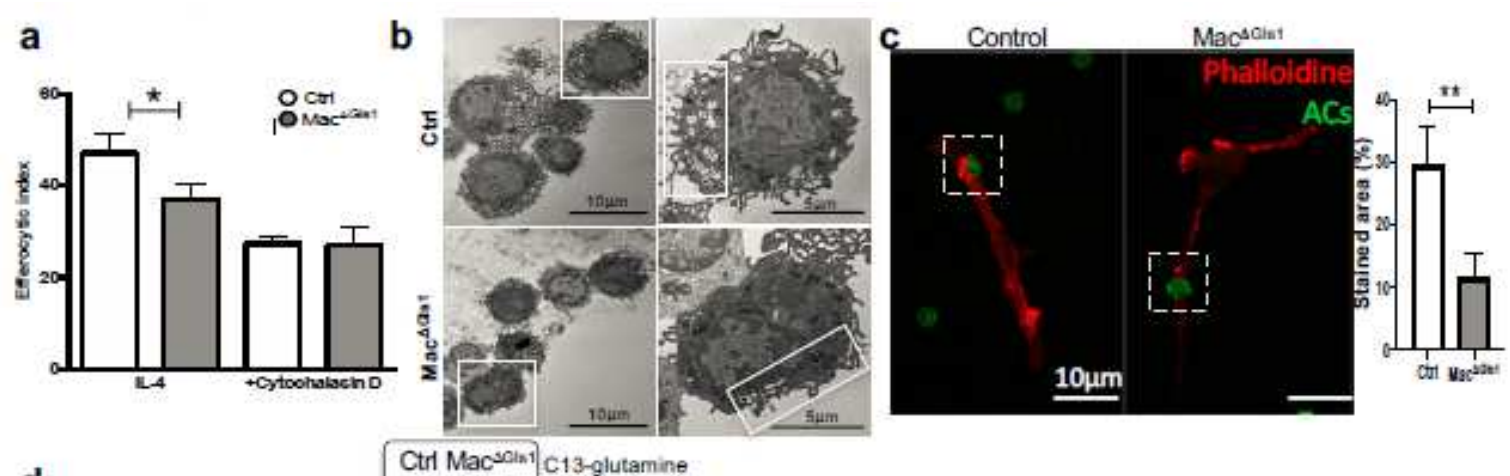

d
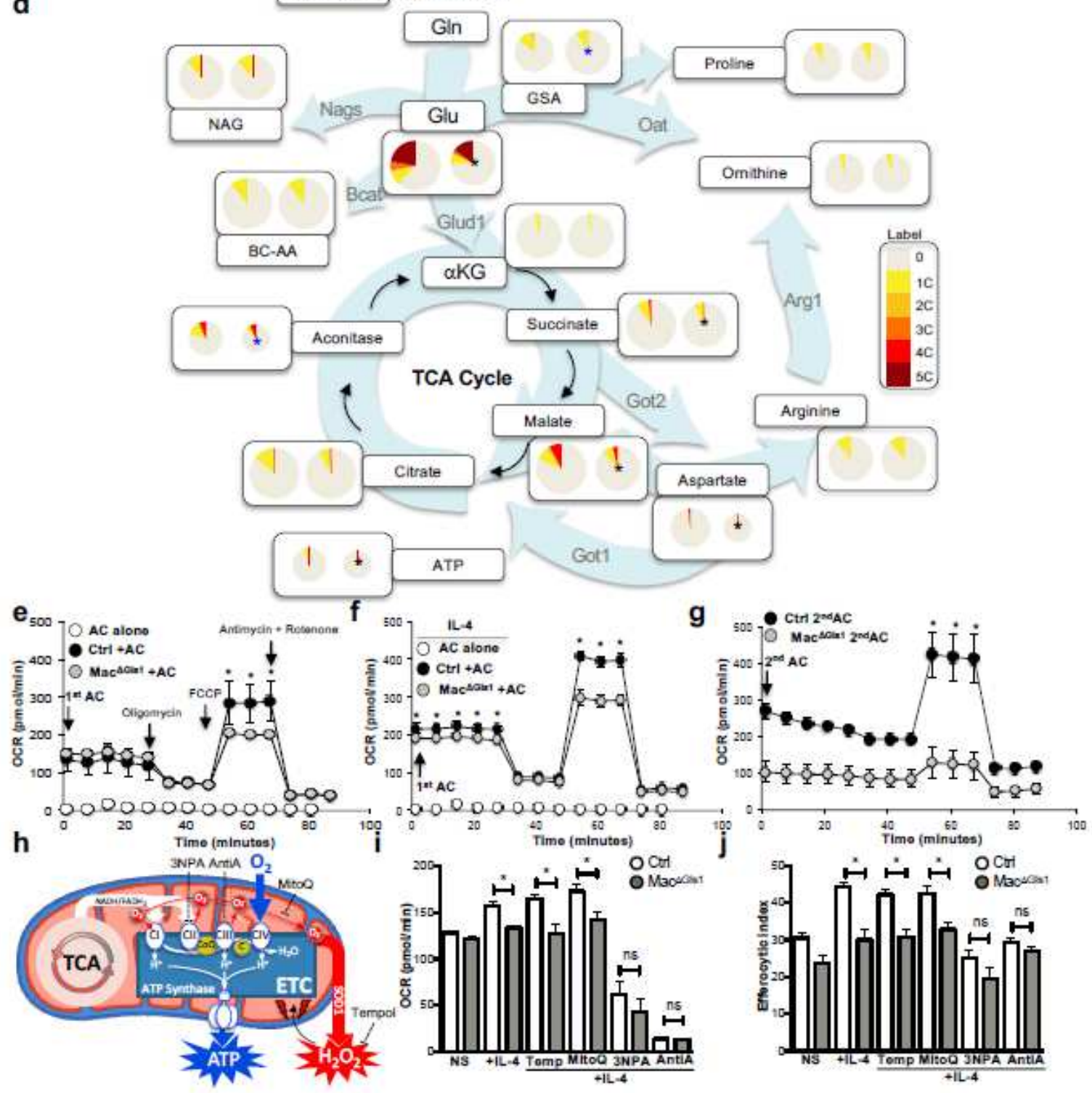

Figure 3

Glutamine metabolism supports the high energy requirement of efferocytosis. (a) Quantification of AC binding and internalization after treatment with $5 \mu \mathrm{M}$ cytochalasin $D$ for $15 \mathrm{~min}$ before the addition of $\mathrm{AC}$ in control or Mac $\Delta$ GIs1 BMDMs stimulated overnight +/- IL-4. (b) Transmission electron microscopy imaging of control or Mac $\Delta \mathrm{Gls} 1 \mathrm{PCMs}$. Left scale bar: $10 \mu \mathrm{m}$. Right scale bar: $5 \mu \mathrm{m}$. (c) Representative images of control or Mac $\Delta$ GIs1 BMDMs stimulated overnight with IL-4 and stained for F-actin (Red) and 
ACs (green). Scale bar: $10 \mu \mathrm{m}$. F-actin staining localized around the phagocytic cup was quantified. (d) Carbon fluxes using U-13C-glutamine. U-13C glutamine was added in the medium of non-stimulated and IL-4-treated macrophages. Circle sizes are scaled with respect to pool size for individual metabolites in each condition. Thin black arrows represent known metabolic pathway connections; background arrows indicate deduced major metabolic flows in alternatively activated macrophages. (e) OCR measured by Seahorse after one incubation with ACs in control or Mac $\triangle$ GIs 1 BMDMs in basal conditions or (f) stimulated overnight with IL-4. (g) OCR measurement after two exposures with ACs in control or Mac $\Delta$ GIs1 BMDMs. (h) Schematic representation of mitochondria electron transport chain and its pharmacological inhibitors. (i) OCR quantification and (j) efferocytic index of control or Mac $\Delta \mathrm{Gls} 1$ BMDMs at steady state or after overnight IL-4 stimulation +/- Tempol, Mitoquinol, 3NPA or antimicyn A. All values are mean \pm SEM and are representative of at least one experiment $(n=3-9)$. ${ }^{*}<<0.05$ compared to control. 
a

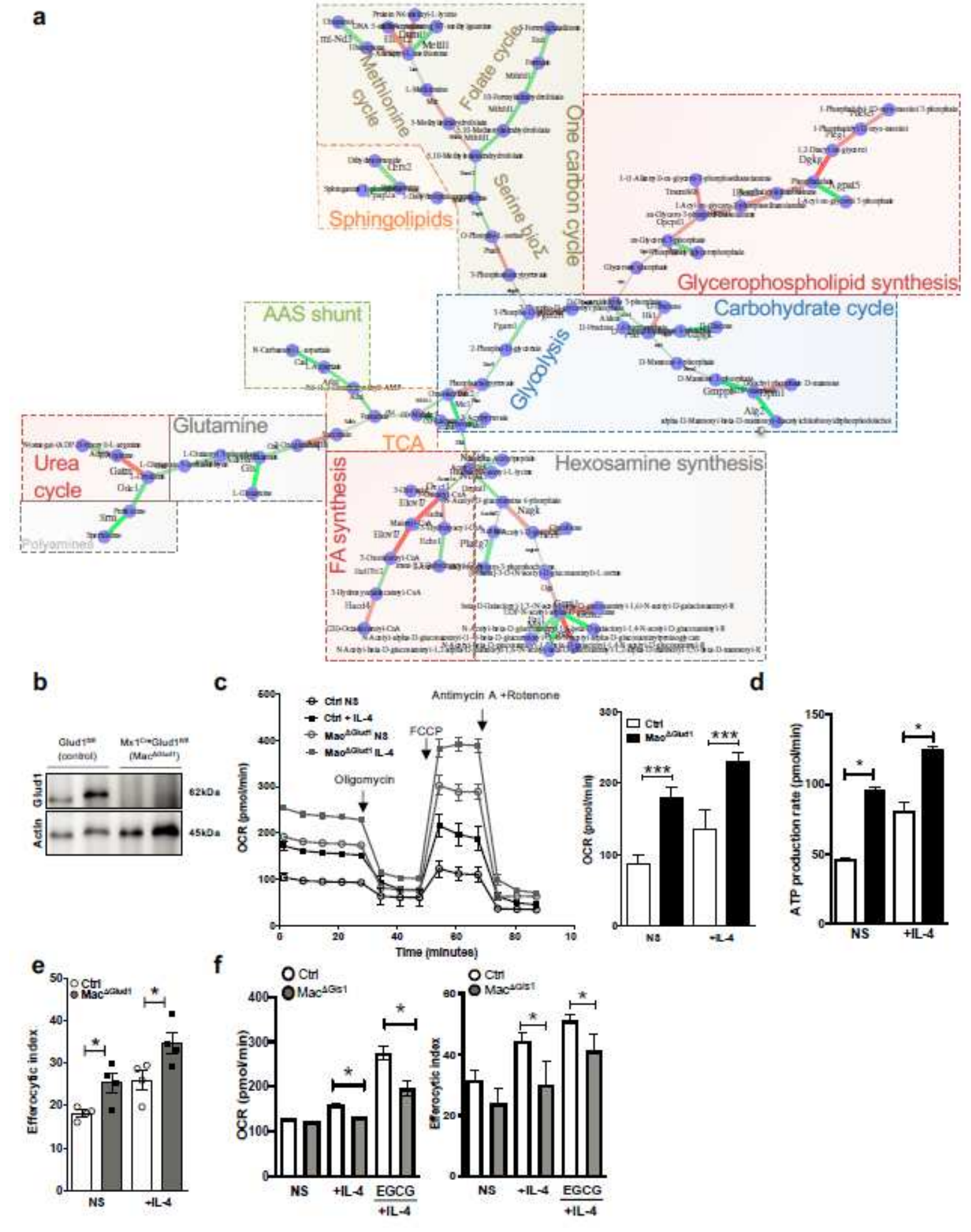

Figure 4

Macrophages utilize a non-canonical glutamine metabolism pathway to promote efficient efferocytosis.

(a) CoMBI-T profiling analysis from RNAseq data of alternatively activated Gls1-deficient or sufficient PCMs. (b) Glud1 protein expression assessed by Western blotting in control or HSCAGlud1 PCMs. (c) OCR and (d) ATP production rate measured by Seahorse in control or Mac $\Delta$ Glud1 BMDMs at steady state or after overnight IL-4 stimulation. (e) efferocytic index in control or Mac $\Delta$ Glud1 BMDMs at steady state or 
after overnight IL-4 stimulation. (f) OCR quantification (left) and efferocytic index (right) from control or MacDGIs1 BMDMs in basal conditions or overnight IL-4 stimulation +/- EGCG. All values are mean \pm SEM and are representative of at least one experiment $(n=3-8) .{ }^{*} P<0.05$, $* * * P<0.0005$ compared to control.
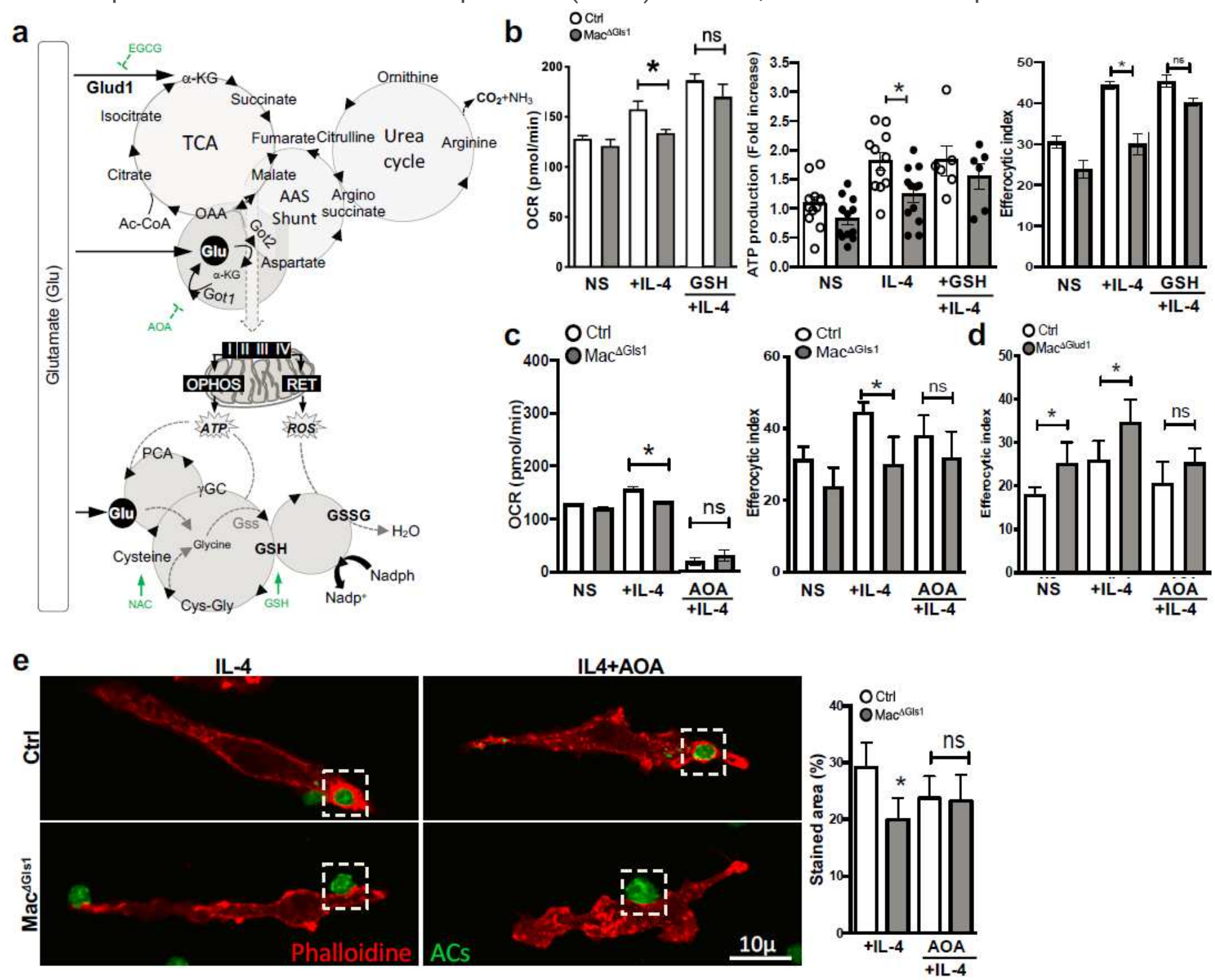

Figure 5

Non-canonical transaminase pathway supports glutamine carbon skeleton metabolism to promote cytoskeletal rearrangement and corpse engulfment. (a) Schematic representation of glutamate incorporation into metabolic cycles. (b) OCR quantification (left), ATP production (middle) and efferocytic index (right) in control or Mac $\triangle$ GIs1 BMDMs in basal conditions or overnight IL-4 stimulation +/- GSH. (c) OCR quantification (left) and efferocytic index (right) in control or Mac $\Delta$ GIs1 BMDMs in basal conditions or overnight IL-4 stimulation +/- AOA. (d) OCR quantification (left) and efferocytic index (right) in control or Mac $\Delta$ Glud1 BMDMs in basal conditions or overnight IL-4 stimulation +/- AOA. (e) Representative images of control or Mac $\triangle \mathrm{Gls} 1 \mathrm{BMDMs}$ stimulated overnight with IL-4 in presence or absence of AOA and

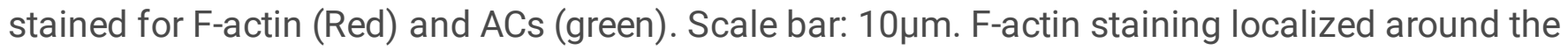


phagocytic cup was quantified. All values are mean \pm SEM and are representative of at least one experiment $(n=3-12)$. ${ }^{2}<0.05$ compared to control. 